\title{
Relative node polynomials for plane curves
}

\author{
Florian Block
}

Received: 14 February 2011 / Accepted: 18 November 2011 / Published online: 13 December 2011

(C) Springer Science+Business Media, LLC 2011

\begin{abstract}
We generalize the recent work of S. Fomin and G. Mikhalkin on polynomial formulas for Severi degrees.

The degree of the Severi variety of plane curves of degree $d$ and $\delta$ nodes is given by a polynomial in $d$, provided $\delta$ is fixed and $d$ is large enough. We extend this result to generalized Severi varieties parametrizing plane curves that, in addition, satisfy tangency conditions of given orders with respect to a given line. We show that the degrees of these varieties, appropriately rescaled, are given by a combinatorially defined "relative node polynomial" in the tangency orders, provided the latter are large enough. We describe a method to compute these polynomials for arbitrary $\delta$, and use it to present explicit formulas for $\delta \leq 6$. We also give a threshold for polynomiality, and compute the first few leading terms for any $\delta$.
\end{abstract}

Keywords Enumerative geometry $\cdot$ Floor diagram $\cdot$ Gromov-Witten theory $\cdot$ Node polynomial $\cdot$ Tangency conditions

\section{Introduction and main results}

The Severi degree $N^{d, \delta}$ is the degree of the Severi variety of (possibly reducible) nodal plane curves of degree $d$ with $\delta$ nodes. Equivalently, $N^{d, \delta}$ is the number of such curves passing through $\frac{(d+3) d}{2}-\delta$ generic points in the complex projective plane $\mathbb{C P}^{2}$. Severi varieties have received considerable attention since they were introduced by F. Enriques [9] and F. Severi [19] around 1915. Much later, in 1986, J. Harris [14] achieved a celebrated breakthrough by showing their irreducibility.

In 1994, P. Di Francesco and C. Itzykson [8] conjectured that the numbers $N^{d, \delta}$ are given by a polynomial in $d$, for a fixed number of nodes $\delta$, provided $d$ is large enough.

F. Block (凶)

Mathematics Institute, University of Warwick, Coventry, CV4 7AL, UK

e-mail: f.s.block@warwick.ac.uk 
S. Fomin and G. Mikhalkin [10, Theorem 5.1] established this polynomiality in 2009. More precisely, they showed that there exists, for every $\delta \geq 1$, a node polynomial $N_{\delta}(d)$ which satisfies $N^{d, \delta}=N_{\delta}(d)$, for all $d \geq 2 \delta$.

The polynomiality of $N^{d, \delta}$ and the polynomials $N_{\delta}(d)$ were known in the 19th century for $\delta=1,2$ and 3 . For $\delta=4,5$ and 6 , this was only achieved by I. Vainsencher [23] in 1995. In 2001, S. Kleiman and R. Piene [15] settled the cases $\delta=7$ and 8. In [3], the author computed $N_{\delta}(d)$ for $\delta \leq 14$ and improved the threshold of S. Fomin and G. Mikhalkin by showing that $N^{d, \delta}=N_{\delta}(d)$ provided $d \geq \delta$.

Severi degrees can be generalized to incorporate tangency conditions to a fixed line $L \subset \mathbb{C P}^{2}$. More specifically, the relative Severi degree $N_{\alpha, \beta}^{\delta}$ is the number of (possibly reducible) nodal plane curves with $\delta$ nodes that have tangency of order $i$ to $L$ at $\alpha_{i}$ fixed points (chosen in advance) and tangency of order $i$ to $L$ at $\beta_{i}$ unconstrained points, for all $i \geq 1$, and that pass through an appropriate number of generic points. Equivalently, $N_{\alpha, \beta}^{\bar{\delta}}$ is the degree of the generalized Severi variety studied in [7, 24]. By Bézout's Theorem, the degree of a curve with tangencies of order $(\alpha, \beta)$ equals $d=\sum_{i \geq 1} i\left(\alpha_{i}+\beta_{i}\right)$. The number of point conditions (for a potentially finite count) is $\frac{(d+3) \bar{d}}{2}-\delta-\alpha_{1}-\alpha_{2}-\cdots$. We recover non-relative Severi degrees by specializing to $\alpha=(0,0, \ldots)$ and $\beta=(d, 0,0, \ldots)$. The numbers $N_{\alpha, \beta}^{\delta}$ are determined by the rather complicated Caporaso-Harris recursion [7].

In this paper, we show that much of the story of (non-relative) node polynomials carries over to relative Severi degrees. Our main result is that, up to a simple combinatorial factor and for fixed $\delta \geq 1$, the relative Severi degrees $N_{\alpha, \beta}^{\delta}$ are given by a multivariate polynomial in $\alpha_{1}, \alpha_{2}, \ldots, \beta_{1}, \beta_{2}, \ldots$, provided that $\beta_{1}+\beta_{2}+\cdots$ is sufficiently large. For a sequence $\alpha=\left(\alpha_{1}, \alpha_{2}, \ldots\right)$ of non-negative integers with only finitely many $\alpha_{i}$ non-zero, we write

$$
|\alpha| \stackrel{\text { def }}{=} \alpha_{1}+\alpha_{2}+\cdots, \quad \alpha ! \stackrel{\text { def }}{=} \alpha_{1} ! \cdot \alpha_{2} ! \cdots
$$

Throughout the paper, we use the grading $\operatorname{deg}\left(\alpha_{i}\right)=\operatorname{deg}\left(\beta_{i}\right)=1$ (so that $d$ and $|\beta|$ are homogeneous of degree 1). The following is our main result.

Theorem 1 For every $\delta \geq 1$, there is a combinatorially defined polynomial $N_{\delta}(\alpha ; \beta)$ in $\alpha_{1}, \alpha_{2}, \ldots, \beta_{1}, \beta_{2}, \ldots$ of (total) degree $3 \delta$ such that, for all $\alpha_{1}, \alpha_{2}, \ldots, \beta_{1}, \beta_{2}, \ldots$ with $|\beta| \geq \delta$, the relative Severi degree $N_{\alpha, \beta}^{\delta}$ is given by

$$
N_{\alpha, \beta}^{\delta}=1^{\beta_{1}} 2^{\beta_{2}} \ldots \frac{(|\beta|-\delta) !}{\beta !} \cdot N_{\delta}\left(\alpha_{1}, \alpha_{2}, \ldots ; \beta_{1}, \beta_{2}, \ldots\right) .
$$

We call $N_{\delta}(\alpha ; \beta)$ the relative node polynomial and use the same notation as in the non-relative case if no confusion can occur. We do not need to specify the number of variables in light of the following stability result.

Theorem 2 For $\delta \geq 1$ and vectors $\alpha=\left(\alpha_{1}, \ldots, \alpha_{m}\right), \beta=\left(\beta_{1}, \ldots, \beta_{m^{\prime}}\right)$ with $|\beta| \geq \delta$, the following polynomial identities hold:

$$
N_{\delta}(\alpha, 0 ; \beta)=N_{\delta}(\alpha ; \beta) \quad \text { and } \quad N_{\delta}(\alpha ; \beta, 0)=N_{\delta}(\alpha ; \beta) .
$$


Therefore, there exists a formal power series $N_{\delta}^{\infty}(\alpha ; \beta)$ in infinitely many variables $\alpha_{1}, \alpha_{2}, \ldots, \beta_{1}, \beta_{2}, \ldots$ that specializes to all relative node polynomials under $\alpha_{m+1}=$ $\alpha_{m+2}=\cdots=0$ and $\beta_{m^{\prime}+1}=\beta_{m^{\prime}+2}=\cdots=0$, for various $m, m^{\prime} \geq 1$.

In fact, even more is true.

Proposition 1 For $\delta \geq 1$, the relative node polynomial $N_{\delta}(\alpha, \beta)$ is a polynomial in $d,|\beta|, \alpha_{1}, \ldots, \alpha_{\delta}$, and $\beta_{1}, \ldots, \beta_{\delta}$, where $d=\sum_{i \geq 1} i\left(\alpha_{i}+\beta_{i}\right)$.

Using the combinatorial description, we provide a method to compute the relative node polynomials for any $\delta$ (see Sects. 3 and 4). We use it to compute $N_{\delta}(\alpha ; \beta)$ for $\delta \leq 6$. Due to spacial constrains, we only tabulate the cases $\delta \leq 3$. The polynomials $N_{0}$ and $N_{1}$ already appeared (implicitly) in [10, Sect. 4.2].

Theorem 3 The relative node polynomials $N_{\delta}(\alpha ; \beta)$, for $\delta=0,1,2,3$ (resp., $\delta \leq 6$ ) are as listed in Appendix (resp., as provided in the ancillary files of the arXiv-version of this paper [2]).

The polynomial $N_{\delta}(\alpha ; \beta)$ is of degree $3 \delta$ by Theorem 1 . We compute the terms of $N_{\delta}(\alpha ; \beta)$ of degree $\geq 3 \delta-2$.

Theorem 4 The terms of $N_{\delta}(\alpha ; \beta)$ of (total) degree $\geq 3 \delta-2$ are given by

$$
\begin{aligned}
N_{\delta}(\alpha ; \beta)= & \frac{3^{\delta}}{\delta !}\left[d^{2 \delta}|\beta|^{\delta}+\frac{\delta}{3}\left[-\frac{3}{2}(\delta-1) d^{2}\right.\right. \\
& \left.-8 d|\beta|+|\beta| \alpha_{1}+d \beta_{1}+|\beta| \beta_{1}\right] d^{2 \delta-2}|\beta|^{\delta-1} \\
& +\frac{\delta}{9}\left[\frac{3}{8}(\delta-1)(\delta-2)(3 \delta-1) d^{4}+12 \delta(\delta-1) d^{3}|\beta|+(11 \delta+1) d^{2}|\beta|^{2}\right. \\
& -\frac{3}{2} \delta(\delta-1)\left(d^{3} \beta_{1}+d^{2}|\beta| \alpha_{1}\right)-\frac{1}{2}(\delta+5)(3 \delta-2) d^{2}|\beta| \beta_{1} \\
& -8(\delta-1)\left(d|\beta|^{2} \alpha_{1}+d|\beta|^{2} \beta_{1}\right)+\frac{1}{2}(\delta-1)\left(d^{2} \beta_{1}^{2}+|\beta|^{2} \alpha_{1}^{2}+|\beta|^{2} \beta_{1}^{2}\right) \\
& \left.\left.+(\delta-1)\left(d|\beta| \alpha_{1} \beta_{1}+d|\beta| \beta_{1}^{2}+|\beta|^{2} \alpha_{1} \beta_{1}\right)\right] d^{2 \delta-4}|\beta|^{\delta-2}+\cdots\right],
\end{aligned}
$$

where $d=\sum_{i \geq 1} i\left(\alpha_{i}+\beta_{i}\right)$.

Theorem 4 can be extended to terms of $N_{\delta}(\alpha, \beta)$ of degree $\geq 3 \delta-7$ (see Remark 3). In Theorem 4, we observe that all coefficients of $N_{\delta}(\alpha ; \beta)$ in degree $\geq 3 \delta-2$ are of the form $\frac{3^{\delta}}{\delta !}$ times a polynomial in $\delta$. Without computing the coefficients, we can extend this further. It is conceivable to expect this property of the coefficients to hold for arbitrary degrees, which, in the special case of non-relative Severi degrees, was shown by N. Qviller [17]. 
Proposition 2 Every coefficient of $N_{\delta}(\alpha ; \beta)$ in degree $\geq 3 \delta-7$ is given, up to a factor of $\frac{3^{\delta}}{\delta !}$, by a polynomial in $\delta$ with rational coefficients.

In 1997, L. Göttsche [12] conjectured universal polynomiality for Severi degrees of smooth polarized projective surfaces $(S, \mathscr{L})$, where $\mathscr{L}$ is an ample line bundle on $S$. More precisely, he conjectured, for any fixed number of nodes, the existence of a universal polynomial that, evaluated at Chern numbers of $(S, \mathscr{L})$, equals the Severi degree of $(S, \mathscr{L})$, provided that $\mathscr{L}$ is sufficiently ample. Göttsche's conjecture was recently proved by the celebrated work of Y.-J. Tzeng [22].

Our approach to planar enumerative geometry is combinatorial and inspired by tropical geometry, in which one replaces a subvariety of a complex algebraic torus by a piecewise linear polyhedral complex (see, for example, $[11,18,20]$ ). By the celebrated Correspondence Theorem of G. Mikhalkin [16, Theorem 1], one can replace the algebraic curve count in $\mathbb{C P}^{2}$ by an enumeration of certain tropical curves. E. Brugallé and G. Mikhalkin [5, 6] introduced a class of decorated graphs, called (marked) floor diagrams (see Sect. 2), which, if weighted correctly, are equinumerous to such tropical curves. We use a version of these results that incorporates tangency conditions due to S. Fomin and G. Mikhalkin [10, Theorem 5]. S. Fomin and G. Mikhalkin also introduced a template decomposition of floor diagrams, which we extend to be suitable for the relative case. This decomposition is crucial in the proofs of all results in this paper, as is the reformulation of algebraic curve counts in terms of floor diagrams.

To the author's knowledge, the polynomiality of the well-studied relative Severi degrees $N_{\alpha, \beta}^{\delta}$ was not expected and came as a surprise to many experts in the field. As the methods in this paper are a natural, somewhat technical extension of those in $[3,10]$, our contribution can also be seen as establishing an unexpected result on enumerative geometry of plane curves, a field with extensive history and the focus of immense recent study.

In related work, F. Ardila and the author [1] generalized the polynomiality of Severi degrees to a family of (in general non-smooth) toric surfaces including $\mathbb{C P}^{1} \times \mathbb{C P}^{1}$ and Hirzebruch surfaces. A main feature is that we showed polynomiality not only in the multi-degree of the curves but also "in the surface itself." In [4], A. Gathmann, H. Markwig and the author defined Psi-floor diagrams that enumerate plane curves that satisfy point and tangency conditions, and conditions given by Psi-classes. We proved a Caporaso-Harris type recursion for Psi-floor diagrams, and showed that relative descendant Gromov-Witten invariants equal their tropical counterparts.

This paper is organized as follows. In Sect. 2, we review the definition of floor diagrams and their markings. In Sect. 3, we introduce a new decomposition of floor diagrams compatible with tangency conditions. In Sect. 4, we prove Theorems 1, 2 and 3 and Proposition 1. In Sect. 5, we prove Theorem 4 and Proposition 2.

\section{Floor diagrams and relative markings}

Floor diagrams are a class of decorated graphs which, if weighted correctly, enumerate plane curves with prescribed properties. They were introduced by E. Brugallé and 
G. Mikhalkin $[5,6]$ in the non-relative case and generalized to the relative setting by S. Fomin and G. Mikhalkin [10]. We begin with a review of the relative setup, following notation of [10] (where floor diagrams are called "labeled floor diagrams").

Definition 1 A floor diagram $\mathscr{D}$ on a vertex set $\{1, \ldots, d\}$ is a directed graph (possibly with multiple edges) with edge weights $w(e) \in \mathbb{Z}_{>0}$ satisfying:

1. The edge directions preserve the vertex order, i.e., for each edge $i \rightarrow j$ of $\mathscr{D}$ we have $i<j$.

2. (Divergence Condition) For each vertex $j$ of $\mathscr{D}$ :

$$
\operatorname{div}(j) \stackrel{\text { def }}{=} \sum_{\substack{\text { edges } e \\ j \stackrel{e}{\rightarrow} k}} w(e)-\sum_{\substack{\text { edges } e \\ i \stackrel{e}{\rightarrow} j}} w(e) \leq 1 .
$$

This means that at every vertex of $\mathscr{D}$ the total weight of the outgoing edges is larger by at most 1 than the total weight of the incoming edges.

The degree of a floor diagram $\mathscr{D}$ is the number of its vertices. $\mathscr{D}$ is connected if its underlying graph is. Note that in [10] floor diagrams are required to be connected. If $\mathscr{D}$ is connected its genus is the genus of the underlying graph (or the first Betti number of the underlying topological space). The cogenus of a connected floor diagram $\mathscr{D}$ of degree $d$ and genus $g$ is given by $\delta(\mathscr{D})=\frac{(d-1)(d-2)}{2}-g$. If $\mathscr{D}$ is not connected, let $d_{1}, d_{2}, \ldots$ and $\delta_{1}, \delta_{2}, \ldots$ be the degrees and cogenera, respectively, of its connected components. The cogenus of $\mathscr{D}$ is $\delta(\mathscr{D})=\sum_{j} \delta_{j}+\sum_{j<j^{\prime}} d_{j} d_{j^{\prime}}$. Via the correspondence between algebraic curves and floor diagrams [6, Theorem 2.5], these notions correspond literally to the respective analogues for algebraic curves. Connectedness corresponds to irreducibility. Lastly, a marked floor diagram $\mathscr{D}$ has multiplicity ${ }^{1}$

$$
\mu(\mathscr{D}) \stackrel{\text { def }}{=} \prod_{\text {edges } e} w(e)^{2} .
$$

We draw floor diagrams using the convention that vertices in increasing order are arranged left to right. Edge weights of 1 are omitted.

Example 1 An example of a floor diagram of degree $d=4$, genus $g=1$, cogenus $\delta=2$, divergences $1,1,0,-2$, and multiplicity $\mu=4$ is drawn below.

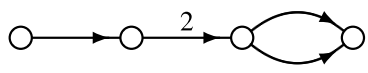

To enumerate algebraic curves satisfying tangency conditions, we need the notion of marked floor diagrams. Our notation, which is more convenient for our purposes, differs slightly from [10], where S. Fomin and G. Mikhalkin define relative markings relative to the partitions $\lambda=\left\langle 1^{\alpha_{1}} 2^{\alpha_{2}} \ldots\right\rangle$ and $\rho=\left\langle 1^{\beta_{1}} 2^{\beta_{2}} \cdots\right\rangle$. In the sequel, all sequences are sequences of non-negative integers with finite support.

\footnotetext{
${ }^{1}$ This agrees with the multiplicity of a tropical plane curve degenerating to a particular floor diagram [10].
} 
Definition 2 For two sequences $\alpha, \beta$ we define an $(\alpha, \beta)$-marking of a floor diagram $\mathscr{D}$ of degree $d=\sum_{i \geq 1} i\left(\alpha_{i}+\beta_{i}\right)$ by the following four step process, which we illustrate in the case of Example 1 for $\alpha=(1,0,0, \ldots)$ and $\beta=(1,1,0,0, \ldots)$.

Step 1: Fix a pair of collections of sequences $\left(\left\{\alpha^{i}\right\},\left\{\beta^{i}\right\}\right)$, where $i$ runs over the vertices of $\mathscr{D}$, such that:

1. The sums over each collection satisfy $\sum_{i=1}^{d} \alpha^{i}=\alpha$ and $\sum_{i=1}^{d} \beta^{i}=\beta$.

2. For all vertices $i$ of $\mathscr{D}$, we have $\sum_{j \geq 1} j\left(\alpha_{j}^{i}+\beta_{j}^{i}\right)=1-\operatorname{div}(i)$.

The second condition says that the "degree of the pair $\left(\alpha^{i}, \beta^{i}\right)$ " is compatible with the divergence at vertex $i$. Each such pair $\left(\left\{\alpha^{i}\right\},\left\{\beta^{i}\right\}\right)$ is called compatible with $\mathscr{D}$ and $(\alpha, \beta)$. We omit writing down trailing zeros.

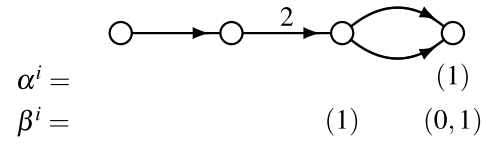

Step 2: For each vertex $i$ of $\mathscr{D}$ and every $j \geq 1$, create $\beta_{j}^{i}$ new vertices, called $\beta$-vertices and illustrated as $\bullet$, and connect them to $i$ with new edges of weight $j$ directed away from $i$. For each vertex $i$ of $\mathscr{D}$ and every $j \geq 1$, create $\alpha_{j}^{i}$ new vertices, called $\alpha$-vertices and illustrated as $\odot$, and connect them to $i$ with new edges of weight $j$ directed away from $i$.

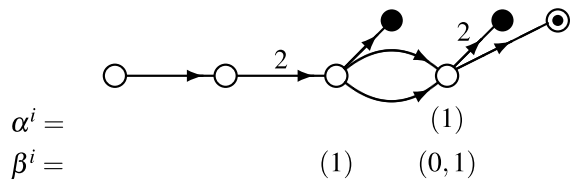

Step 3: Subdivide each edge of the original floor diagram $\mathscr{D}$ into two directed edges by introducing a new vertex for each edge. The new edges inherit their weights and orientations. Call the resulting graph $\tilde{\mathscr{D}}$.

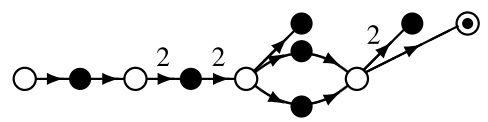

Step 4: Linearly order the vertices of $\tilde{\mathscr{D}}$ extending the order of the vertices of the original floor diagram $\mathscr{D}$ such that, as in $\mathscr{D}$, each edge is directed from a smaller vertex to a larger vertex. Furthermore, we require that the $\alpha$-vertices are largest among all vertices, and for every pair of $\alpha$-vertices $i^{\prime}>i$, the weight of the $i^{\prime}$-adjacent edge is larger than or equal to the weight of the $i$-adjacent edge.

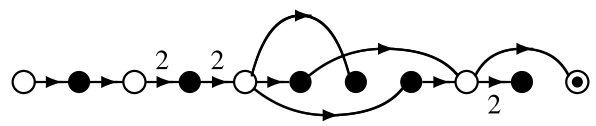


We call the extended graph $\tilde{\mathscr{D}}$, together with the linear order on its vertices, an $(\alpha, \beta)$-marked floor diagram, or an $(\alpha, \beta)$-marking of the floor diagram $\mathscr{D}$.

We need to count $(\alpha, \beta)$-marked floor diagrams up to equivalence. Two $(\alpha, \beta)$ markings $\tilde{\mathscr{D}}_{1}, \tilde{\mathscr{D}}_{2}$ of a floor diagram $\mathscr{D}$ are equivalent if there exists a weight preserving automorphism of weighted graphs mapping $\tilde{\mathscr{D}}_{1}$ to $\tilde{\mathscr{D}}_{2}$ that fixes the vertices of $\mathscr{D}$. The number of markings $v_{\alpha, \beta}(\mathscr{D})$ is the number of $(\alpha, \beta)$-markings of $\mathscr{D}$, up to equivalence. Furthermore, we write $\mu_{\beta}(\mathscr{D})$ for the product $1^{\beta_{1}} 2^{\beta_{2}} \cdots \mu(\mathscr{D})$. In the example in Definition 2, the particular choice of compatible sequences results in a choice of 5 non-equivalent markings in Step 4, thus contributing 5 to $v_{\alpha, \beta}(\mathscr{D})$.

The next theorem follows from [10, Theorem 3.18] by a straightforward extension of the inclusion-exclusion procedure of [10, Sect. 1] that was used to conclude [10, Corollary 1.9] (the non-relative count of reducible curves via floor diagrams) from [10, Theorem 1.6] (the non-relative count of irreducible curves via floor diagrams).

Theorem 5 For any $\delta \geq 1$, the relative Severi degree $N_{\alpha, \beta}^{\delta}$ is given by

$$
N_{\alpha, \beta}^{\delta}=\sum_{\mathscr{D}} \mu_{\beta}(\mathscr{D}) v_{\alpha, \beta}(\mathscr{D})
$$

where the sum is over all (possibly disconnected) floor diagrams $\mathscr{D}$ of degree $d=$ $\sum_{i \geq 1} i\left(\alpha_{i}+\beta_{i}\right)$ and cogenus $\delta$.

\section{Relative decomposition of floor diagrams}

In this section, we introduce a new decomposition of floor diagrams compatible with tangency conditions, which we use extensively in Sects. 4 and 5 to prove all our results stated in Sect. 1. This decomposition is a generalization of ideas of S. Fomin and G. Mikhalkin [10]. We start out by reviewing their key gadget.

Definition 3 A template $\Gamma$ is a directed graph (possibly with multiple edges) on vertices $\{0, \ldots, l\}$, where $l \geq 1$, with edge weights $w(e) \in \mathbb{Z}_{>0}$, satisfying:

1. If $i \rightarrow j$ is an edge, then $i<j$.

2. Every edge $i \stackrel{e}{\rightarrow} i+1$ has weight $w(e) \geq 2$. (No "short edges.")

3. For each vertex $j, 1 \leq j \leq l-1$, there is an edge "covering" it, i.e., there exists an edge $i \rightarrow k$ with $i<j<k$.

Every template $\Gamma$ comes equipped with some natural numerical invariants. Its length $l(\Gamma)$ is the number of vertices minus 1 . The product of squares of the edge weights is its multiplicity $\mu(\Gamma)$. Its cogenus $\delta(\Gamma)$ is

$$
\delta(\Gamma) \stackrel{\text { def }}{=} \sum_{\substack{e \\ i \rightarrow j}}[(j-i) w(e)-1] .
$$




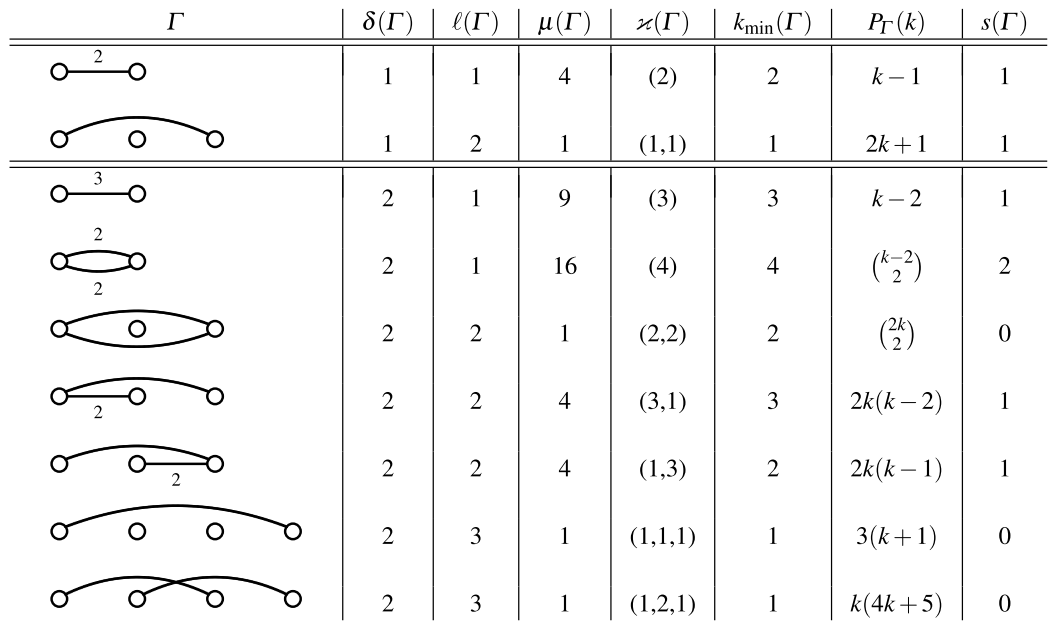

Fig. 1 The templates with $\delta(\Gamma) \leq 2$

Thus, every edge of $\Gamma$ contributes by the product of its length and weight minus 1 to $\delta(\Gamma)$.

For $1 \leq j \leq l(\Gamma)$, let $\varkappa_{j}=\varkappa_{j}(\Gamma)$ denote the sum of the weights of edges $i \stackrel{e}{\rightarrow} k$ with $i<j \leq k$, i.e., $\varkappa_{j}(\Gamma)$ is the sum of the weights of all edges of $\Gamma$ from a vertex left of or equal to $j-1$ to a vertex right of or equal to $j$. We define

$$
k_{\min }(\Gamma) \stackrel{\text { def }}{=} \max _{1 \leq j \leq l}\left(\varkappa_{j}-j+1\right) .
$$

This makes $k_{\min }(\Gamma)$ the smallest positive integer $k$ such that $\Gamma$ can appear in a floor diagram on $\{1,2, \ldots\}$ as a subgraph with left-most vertex $k$. Figure 1 [10, Fig. 10] lists all templates $\Gamma$ with $\delta(\Gamma) \leq 2$.

We now explain how to decompose a floor diagram $\mathscr{D}$ into a collection of templates and further building blocks. The decomposition depends on tangency sequences $\alpha$ and $\beta$ as well as a pair $\left(\left\{\alpha^{i}\right\},\left\{\beta^{i}\right\}\right.$ ) compatible with $\mathscr{D}$ (see Step 1 of Definition 2), where $i$ runs over the vertices of $\mathscr{D}$.

Assume we are given such data $\left(\mathscr{D},\left\{\alpha^{i}\right\},\left\{\beta^{i}\right\}\right)$, and let $d$ be the degree of $\mathscr{D}$. We first construct two (infinite) matrices $A$ and $B$ : for $i \geq 1$, we define the $i$ th row $a_{i}$ resp. $b_{i}$ of $A$ resp. $B$ to be the sequence $\alpha^{d-i}$ resp. $\beta^{d-i}$. (If $d-i \leq 0$, i.e., if $i \geq d$, we set $a_{i}=b_{i}=(0,0, \ldots)$.) This records the sequences $\alpha^{d-1}$ resp. $\beta^{d-1}$ of the second to last vertex of $\mathscr{D}$ in the first row of $A$ resp. $B$, the sequences $\alpha^{d-2}$ resp. $\beta^{d-2}$ associated with the third to last vertex of $\mathscr{D}$ in the second row of $A$ resp. $B$, and so on. Notice that we do not record in $A$ and $B$ the sequences $\alpha^{d}$ and $\beta^{d}$ associated with the right-most vertex of $\mathscr{D}$. These sequences satisfy

$$
\alpha^{d}=\alpha-\sum_{i \geq 1} a_{i} \quad \text { and } \quad \beta^{d}=\beta-\sum_{i \geq 1} b_{i}
$$


and can thus be recovered from $\alpha, \beta, A$ and $B$. Before we continue to describe the decomposition of a floor diagram into templates, we illustrate the previous construction by an example.

Example 2 The pictured pair of sequences $\left(\left\{\alpha^{i}\right\},\left\{\beta^{i}\right\}\right)$ (we omit to write down zerosequences), compatible with the floor diagram $\mathscr{D}$ and $\alpha=(0,1), \beta=(4,1)$,

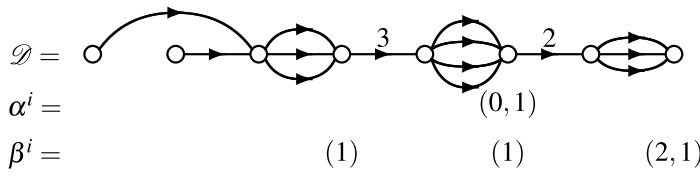

determines the matrices

$$
A=\left[\begin{array}{cccc}
0 & 0 & 0 & \cdots \\
0 & 1 & 0 & \cdots \\
0 & 0 & 0 & \cdots \\
0 & 0 & 0 & \cdots \\
0 & 0 & 0 & \cdots \\
\vdots & \vdots & \vdots & \ddots
\end{array}\right] \text { and } B=\left[\begin{array}{cccc}
0 & 0 & 0 & \cdots \\
1 & 0 & 0 & \cdots \\
0 & 0 & 0 & \cdots \\
1 & 0 & 0 & \ldots \\
0 & 0 & 0 & \cdots \\
\vdots & \vdots & \vdots & \ddots
\end{array}\right]
$$

Next, we describe how the triple $(\mathscr{D}, A, B)$, in turn, determines a collection of templates, together with some extra data. Let $l(A)$ resp. $l(B)$ be the largest row indices such that $A$ resp. $B$ have a non-zero entry in this row. We call these numbers the length of $A$ and $B$. (The length of the zero-matrix is 0 .) After we remove all "short edges" from $\mathscr{D}$, i.e., all edges of weight 1 between consecutive vertices, the resulting graph is an ordered collection of templates $\left(\Gamma_{1}, \ldots, \Gamma_{r}\right)$, listed left to right. Let $k_{s}$ be the smallest vertex in $\mathscr{D}$ of each template $\Gamma_{s}$. Record all pairs $\left(\Gamma_{s}, k_{s}\right)$ that satisfy $k_{s}+l\left(\Gamma_{S}\right) \leq d-\max (l(A), l(B))$, i.e., all templates whose right-most vertex is left of or equal to every vertex $i$ of $\mathscr{D}$ for which $A$ or $B$ have row $d-i$ non-zero. Record the remaining templates, together with all vertices $i$, for $i \geq \max (l(A), l(B))$, in one graph $\Lambda$ on vertices $0, \ldots, l$ by shifting the vertex labels by $d-l$. See Example 3 for an example of this decomposition.

Example 3 The decomposition of the floor diagram $\mathscr{D}$ of Example 2 subject to the matrices $A$ and $B$ of Example 2 is pictured below. Notice that $\max (l(A), l(B))=4$, thus $\Lambda$ contains both the edges of weight 2 and 3 .

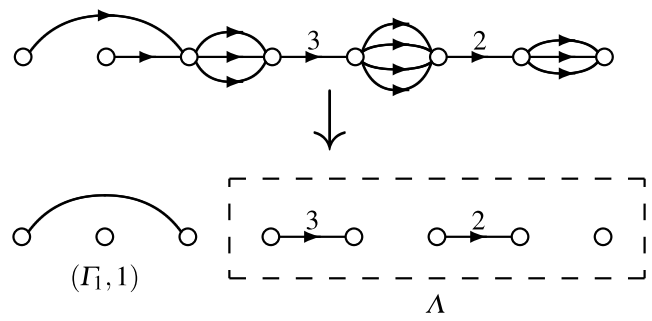


The triple $(\Lambda, A, B)$ in the example above is an instance of an "extended template," a new building block allowing relative decomposition.

Definition $4 \mathrm{~A}$ tuple $(\Lambda, A, B)$ is an extended template of length $l=l(\Lambda)=$ $l(\Lambda, A, B)$ if $\Lambda$ is a directed graph (possibly with multiple edges) on vertices $\{0, \ldots, l\}$, where $l \geq 0$, with edge weights $w(e) \in \mathbb{Z}_{>0}$, satisfying:

1. If $i \rightarrow j$ is an edge then $i<j$.

2. Every edge $i \stackrel{e}{\rightarrow} i+1$ has weight $w(e) \geq 2$. (No "short edges.")

Moreover, $A$ and $B$ are (infinite) matrices with non-negative integral entries and finite support, and we write $l(A)$ and $l(B)$ for the respective largest row indices of $A$ and $B$ of a non-zero entry. Additionally, we demand $l(\Lambda) \geq \max (l(A), l(B))$, and that, for each $1 \leq j \leq l-\max (l(A), l(B))$, there is an edge $i \rightarrow k$ of $\Lambda$ with $i<j<k$.

The last condition in the definition is a kind of "connectedness" property: it says that, for each vertex $j \geq 1$ of $\Lambda$ at least at distance $\max (l(A), l(B))$ from the rightmost vertex $l$ of $\Lambda$, there must be an edge of $\Lambda$ that passes it. This condition implies that once $l(\Lambda) \geq 1$, the matrices $A$ and $B$ cannot both be the zero-matrix.

From a floor diagram $\mathscr{D}$, sequences $\alpha$ and $\beta$, and a compatible pair $\left(\left\{\alpha^{i}\right\},\left\{\beta^{i}\right\}\right)$, we have constructed two successive maps:

$$
\left(\mathscr{D},\left(\alpha^{i}\right),\left(\beta^{i}\right)\right) \rightarrow(\mathscr{D}, A, B) \rightarrow\left(\left\{\left(\Gamma_{s}, k_{s}\right)\right\}, \Lambda, A, B\right)
$$

The two maps are illustrated in Examples 2 and 3, respectively.

We now analyze when these maps are reversible. Fix a collection $\left\{\left(\Gamma_{s}, k_{s}\right)\right\}$ of templates and positive integers, where $1 \leq s \leq m$, an extended template $(\Lambda, A, B)$, and a positive integer $d$ (which will be the degree of $\mathscr{D}$ ). Then the second map of (3) is reversible if and only if

$$
\begin{cases}k_{i} \geq k_{\min }\left(\Gamma_{i}\right) & \text { for } 1 \leq i \leq m, \\ k_{i+1} \geq k_{i}+l\left(\Gamma_{i}\right) & \text { for } 1 \leq i \leq m-1, \\ k_{m}+l\left(\Gamma_{m}\right) \leq d-l(\Lambda) . & \end{cases}
$$

In other words, the templates cannot appear too far to the left, and the graphs $\Gamma_{1}, \ldots, \Gamma_{m}$ and $\Lambda$ cannot overlap.

Whether the first map is reversible depends on the sequences $\alpha$ and $\beta$. Recall that, given a compatible pair $\left(\left\{\alpha^{i}\right\},\left\{\beta^{i}\right\}\right)$, we only recorded in the matrices $A$ and $B$ the pairs $\left(\alpha^{i}, \beta^{i}\right)$ for $i<d$. The pair $\left(\alpha^{d}, \beta^{d}\right)$, in turn, is determined by (2). As the entries of $\alpha^{d}$ and $\beta^{d}$ represent numbers of edges we need to add at vertex $d$ to obtain an $(\alpha, \beta)$-marking of $\mathscr{D}$ (see Step 2 of Definition 2), all entries of $\alpha^{d}$ and $\beta^{d}$ need to be non-negative. Thus, the first map in (3) is reversible if and only if component-wise

$$
\sum_{i \geq 1} a_{i} \leq \alpha \quad \text { and } \quad \sum_{i \geq 1} b_{i} \leq \beta .
$$

It follows that, for large enough $\alpha$ and $\beta$, the decomposition is independent of $\alpha$ and $\beta$, given the part of the compatible pair $\left(\left\{\alpha^{i}\right\},\left\{\beta^{i}\right\}\right)$, for $1 \leq i<d$, away from 
the right-most vertex of $\mathscr{D}$. It is exactly this part that is recorded by the matrices $A$ and $B$. Thus, we can think of $A$ and $B$ as sort of "placeholders" for $\alpha^{i}$ and $\beta^{i}$, in the case where $\alpha$ and $\beta$ varies.

Example 4 We now illustrate the reverse direction of (3), that is, how to build up a floor diagram (and a compatible pair of sequences) from the pieces of the decomposition. Let $\Gamma_{1}, \Gamma_{2}$ and $\Lambda$ be the three graphs and $A$ and $B$ be the two matrices below.

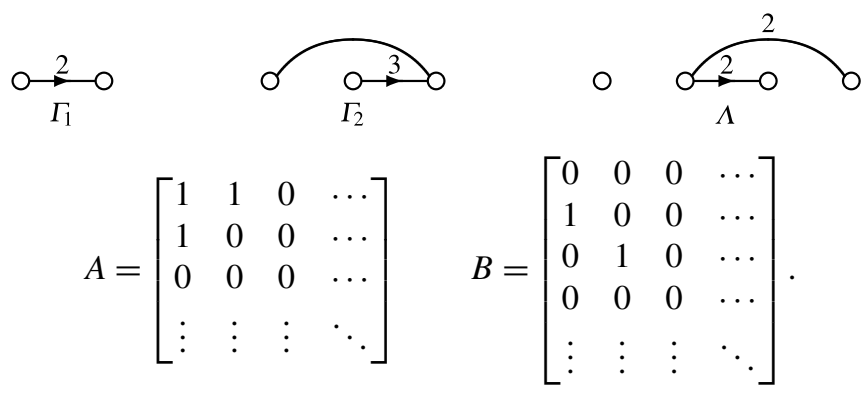

Notice that, without the left-most vertex in $\Lambda$, the triple $(\Lambda, A, B)$ is not an extended template (otherwise, $l(\Lambda)=2$ although $l(B)=3$, as $B$ has non-trivial third row).

Let $\alpha$ and $\beta$ be sufficiently large tangency sequences satisfying (5). The degree of the floor diagram $\mathscr{D}$ we want to construct is $d=\sum_{i} i\left(\alpha_{i}+\beta_{i}\right)$ (following Bézout's Theorem). We need to choose the "positions" $k_{1}$ and $k_{2}$ of the templates $\Gamma_{1}$ and $\Gamma_{2}$ in accordance with (4). An invalid choice is $k_{1}=1$, as it violates $k_{1} \geq k_{\min }\left(\Gamma_{1}\right)=2$. This is reflecting the fact that the divergence condition of floor diagrams forbids weight- 2 edges adjacent to the first vertex.

A valid choice, however, is $k_{1}=2$ and $k_{2}=3$, as for $d$ large enough, $k_{1}$ and $k_{2}$ satisfy (5). From $A$ and $B$ we can directly read off $\alpha^{i}$ and $\beta^{i}$ for $i<d$ and determine the floor diagram $\mathscr{D}$ : between each pair of adjacent vertices $i$ and $i+1$, we need to add sufficiently many edges of length 1 and weight 1 (the "short edges"), so that, after adding the $\alpha$-and $\beta$-edges of a marking of $\mathscr{D}$, the total weight of the edges from vertices left of or equal to $i$ to vertices right of or equal to $i+1$ is $i$. This makes the divergence (i.e., "outflow minus inflow") of each vertex of $\mathscr{D}$ in the marked floor diagram equal 1 . For a formula of the necessary number of short edges see (7) and (8) below. To illustrate, if $\left(\mathscr{D},\left\{\alpha^{i}\right\},\left\{\beta^{i}\right\}\right)$ is

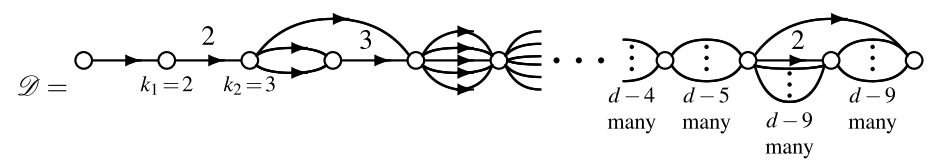

$$
\begin{aligned}
& \alpha^{i}= \\
& \beta^{i}=
\end{aligned}
$$

(1) $(1,1)$

$$
\begin{aligned}
& \alpha^{d} \\
& \beta^{d}
\end{aligned}
$$

we need to add $d-5$ short edges between the third-last and second-last vertex of $\mathscr{D}$ : each marking $\tilde{\mathscr{D}}$ of $\mathscr{D}$ has one $\beta$-edge of weight 2 emerging from the third-last vertex (as $\left.\beta^{d-3}=(0,1)\right)$, and we want the total weight of edges of $\tilde{\mathscr{D}}$ between vertices left of or equal to vertex $d-3$ and right of or equal to vertex $d-2$ to equal $d-3$. 


\begin{tabular}{|c|c|c|c|c|c|c|c|c|c|c|}
\hline \multicolumn{4}{|c|}{$(\Lambda, A, B)$} & $\delta$ & $l$ & $\mu$ & $\varkappa$ & $d_{\min }$ & $q_{(\Lambda, A, B)}(\alpha ; \beta)$ of Lemma 1 & $s$ \\
\hline O & & & {$\left[\begin{array}{ll}0 & 0 \\
0 & 0\end{array}\right]\left[\begin{array}{ll}0 & 0 \\
0 & 0\end{array}\right]$} & 0 & 0 & 1 & () & 1 & 1 & 0 \\
\hline O & O & & {$\left[\begin{array}{ll}1 & 0 \\
0 & 0\end{array}\right]\left[\begin{array}{ll}0 & 0 \\
0 & 0\end{array}\right]$} & 1 & 1 & 1 & (0) & 1 & 1 & 0 \\
\hline 0 & O & & {$\left[\begin{array}{ll}0 & 0 \\
0 & 0\end{array}\right]\left[\begin{array}{ll}1 & 0 \\
0 & 0\end{array}\right]$} & 1 & 1 & 1 & (0) & 1 & $\beta_{1}(d+|\beta|-1)$ & 0 \\
\hline o- & - & & {$\left[\begin{array}{ll}1 & 0 \\
0 & 0\end{array}\right]\left[\begin{array}{ll}0 & 0 \\
0 & 0\end{array}\right]$} & 2 & 1 & 4 & (2) & 4 & $(d-3)$ & 1 \\
\hline o- & -0 & & {$\left[\begin{array}{ll}0 & 0 \\
0 & 0\end{array}\right]\left[\begin{array}{ll}1 & 0 \\
0 & 0\end{array}\right]$} & 2 & 1 & 4 & (2) & 4 & $\beta_{1}(d-3)(d+|\beta|-2)$ & 1 \\
\hline$\sigma$ & 0 & r & {$\left[\begin{array}{ll}1 & 0 \\
0 & 0\end{array}\right]\left[\begin{array}{ll}0 & 0 \\
0 & 0\end{array}\right]$} & 2 & 2 & 1 & $(1,1)$ & 3 & $2(d-2)$ & 0 \\
\hline o & 0 & ○ & {$\left[\begin{array}{ll}0 & 0 \\
0 & 0\end{array}\right]\left[\begin{array}{ll}1 & 0 \\
0 & 0\end{array}\right]$} & 2 & 2 & 1 & $(1,1)$ & 3 & $\beta_{1}(d-2)(2 d+2|\beta|-3)$ & 0 \\
\hline O & ० & & {$\left[\begin{array}{ll}2 & 0 \\
0 & 0\end{array}\right]\left[\begin{array}{ll}0 & 0 \\
0 & 0\end{array}\right]$} & 2 & 1 & 1 & (0) & 3 & 1 & 0 \\
\hline O & 0 & & {$\left[\begin{array}{ll}1 & 0 \\
0 & 0\end{array}\right]\left[\begin{array}{ll}1 & 0 \\
0 & 0\end{array}\right]$} & 2 & 1 & 1 & (0) & 3 & $\beta_{1}(d+|\beta|-2)$ & 0 \\
\hline O & ० & & {$\left[\begin{array}{ll}0 & 0 \\
0 & 0\end{array}\right]\left[\begin{array}{ll}2 & 0 \\
0 & 0\end{array}\right]$} & 2 & 1 & 1 & (0) & 3 & $\left(\begin{array}{c}\beta_{1} \\
2\end{array}\right)\left(d^{2}+2 d|\beta|+|\beta|^{2}-5 d-5|\beta|+6\right)$ & 0 \\
\hline O & 0 & & {$\left[\begin{array}{ll}0 & 1 \\
0 & 0\end{array}\right]\left[\begin{array}{ll}0 & 0 \\
0 & 0\end{array}\right]$} & 2 & 1 & 1 & (0) & 3 & 1 & 0 \\
\hline 0 & 0 & & {$\left[\begin{array}{ll}0 & 0 \\
0 & 0\end{array}\right]\left[\begin{array}{ll}0 & 1 \\
0 & 0\end{array}\right]$} & 2 & 1 & 1 & (0) & 3 & $\beta_{2}(|\beta|-1)(d+|\beta|-2)$ & 0 \\
\hline 0 & 0 & 0 & {$\left[\begin{array}{ll}0 & 0 \\
1 & 0\end{array}\right]\left[\begin{array}{ll}0 & 0 \\
0 & 0\end{array}\right]$} & 2 & 3 & 1 & $(0,0)$ & 3 & 1 & 0 \\
\hline 0 & 0 & 0 & {$\left[\begin{array}{ll}0 & 0 \\
0 & 0\end{array}\right]\left[\begin{array}{ll}0 & 0 \\
1 & 0\end{array}\right]$} & 2 & 3 & 1 & $(0,0)$ & 3 & $\beta_{1}(|\beta|-1)(2 d+|\beta|-3)$ & 0 \\
\hline
\end{tabular}

Fig. 2 The extended templates with $\delta(\Lambda, A, B) \leq 2$

The sequences $\alpha^{d}$ and $\beta^{d}$ of the right-most vertex of $\mathscr{D}$ are functions of the tangency sequences $\alpha$ and $\beta$. By (2), we have $\alpha^{d}=\left(\alpha_{1}-2, \alpha_{2}-1, \alpha_{3}, \alpha_{4}, \ldots\right)$ and $\beta^{d}=\left(\beta_{1}, \beta_{2}-1, \beta_{3}-1, \beta_{4}, \ldots\right)$. Thus, the inverse map of (3) is defined provided $\alpha_{1} \geq 2, \alpha_{2}, \beta_{2}, \beta_{3} \geq 1$, and $\beta_{1}, \alpha_{3}, \alpha_{i}, \beta_{i} \geq 0$, for $i \geq 4$. This concludes the example.

The cogenus $\delta(\Lambda, A, B)$ of an extended template $(\Lambda, A, B)$ is the sum of the cogenera $\delta(\Lambda), \delta(A)$ and $\delta(B)$, where

$$
\delta(\Lambda) \stackrel{\text { def }}{=} \sum_{\substack{e \\ i \rightarrow j}}((j-i) w(e)-1) \quad \text { and } \quad \delta(A) \stackrel{\text { def }}{=} \sum_{i, j \geq 1} i \cdot j \cdot a_{i, j},
$$

and similarly for $B$. Figure 2 shows all extended templates with cogenus at most 2 . This list can be obtained by first considering all pairs of matrices $(A, B)$ with $\delta(A)+\delta(B) \leq 2$, and then possibly adding weighted edges such that the last ("connectedness") condition in Definition 4 is still satisfied. The definition of $\delta(\Lambda, A, B)$ is such that the correspondence (3) is cogenus-preserving, in the following sense. 
Proposition 3 Fix two tangency sequences $\alpha$ and $\beta$. Let $\mathscr{D}$ be a floor diagram of degree $d=\sum_{i \geq 1} i\left(\alpha_{i}+\beta_{i}\right)$. Then we have, for each pair $\left(\left\{\alpha^{i}\right\},\left\{\beta^{i}\right\}\right)$ compatible with $\mathscr{D}$ and $(\alpha, \beta)$,

$$
\delta(\mathscr{D})=\left(\sum_{i=1}^{m} \delta\left(\Gamma_{i}\right)\right)+\delta(\Lambda)+\delta(A)+\delta(B),
$$

where $\Gamma_{1}, \ldots, \Gamma_{m}, \Lambda, A$ and $B$ are defined via (3).

Proof First, assume that $\mathscr{D}$ is connected with genus $g(\mathscr{D})$. Then, by definition, $\delta(\mathscr{D})=\frac{(d-1)(d-2)}{2}-g(\mathscr{D})$ is the "genus-deficiency" of $\mathscr{D}$, with respect to the unique template $\mathscr{D}_{0}$ of degree $d$ and genus $\frac{(d-1)(d-2)}{2}$ (so $\delta\left(\mathscr{D}_{0}\right)=0$ ). Specifically, $\mathscr{D}_{0}$ has $i$ edges of weight 1 between vertices $i$ and $i+1$, for $1 \leq i<d$, and no other edges. Let $\Gamma_{1}, \ldots, \Gamma_{m}, \Lambda, A$ and $B$ be the decomposition data of $\mathscr{D}$ according to (3). We will describe a degeneration of $\mathscr{D}$ to $\mathscr{D}_{0}$ which, at each stage, is cogenus-preserving.

If $\Lambda$ has an edge, say $i \stackrel{e}{\rightarrow} j$, let $\Lambda^{\prime}$ be obtained from $\Lambda$ by removing the edge $e$. The floor diagram $\mathscr{D}^{\prime}$ corresponding to the altered data, with $\Lambda^{\prime}$ instead of $\Lambda$, differs from $\mathscr{D}$ by $\operatorname{wt}(e)$ many short edges between each adjacent pair of the len $(e)+1$ many adjacent vertices in $\mathscr{D}^{\prime}$ between $i$ and $j$, where len $(e)=j-i$ is the length of $e$. This alteration increases the genus by $\operatorname{wt}(e) \operatorname{len}(e)-1$, which agrees with the weight of the edge $e$ in the definition of $\delta(\Lambda)$. Thus, we can assume that $\Lambda$ has no edges, and, by the same argument applied to the templates $\Gamma_{i}$, that $m=0$.

If $B$ has a non-zero entry $b_{i^{\prime} j^{\prime}}$, let $B^{\prime}$ be the matrix obtained from $B$ by lowering its $\left(i^{\prime}, j^{\prime}\right)$ th entry by one. Again, let $\mathscr{D}^{\prime}$ be the floor diagram that corresponds to the altered data. Then $\mathscr{D}^{\prime}$ differs from $\mathscr{D}$ by $j^{\prime}$ additional short edges between each adjacent pair of vertices in $\mathscr{D}^{\prime}$ between $d-i^{\prime}$ and $d$, increasing the genus by $i^{\prime} \cdot j^{\prime}$. This agrees with the weight of the $\left(i^{\prime}, j^{\prime}\right)$ th entry of $B$ in the definition of $\delta(B)$, and we can assume that $B$ is zero. Similarly, we can assume that $A$ is zero. Thus, if $\mathscr{D}$ is connected, we are done as the proposition holds for $\mathscr{D}_{0}$.

If $\mathscr{D}$ equals a union $\mathscr{D}_{1} \sqcup \mathscr{D}_{2}$ of two connected floor diagrams $\mathscr{D}_{1}$ and $\mathscr{D}_{2}$, let $\tilde{\mathscr{D}}$ be an $(\alpha, \beta)$-marking of $\mathscr{D}$. To prove the proposition for non-connected floor diagrams, we use the correspondence between marked floor diagrams and tropical plane curves $^{2}$ through a fixed "horizontally stretched" point configuration. ${ }^{3}$ All relevant definitions can, for example, be found in [10, Sects. 2 and 3]. For an illustration of the correspondence, see Fig. 3.

We can identify the marked floor diagram $\tilde{\mathscr{D}}$ with a tropical plane curve $C$ through a horizontally stretched point configuration in $\mathbb{R}^{2}$ (see [10, Theorem 3.17]). The two connected components $\tilde{\mathscr{D}}_{1}$ and $\tilde{\mathscr{D}}_{2}$ of $\tilde{\mathscr{D}}$ are markings of $\mathscr{D}_{1}$ and $\mathscr{D}_{2}$, respectively. Each $\tilde{\mathscr{D}}_{i}$ corresponds to an irreducible component $C_{i}$ of $C$ (i.e., each $C_{i}$ is not a non-trivial union of tropical plane curves).

\footnotetext{
${ }^{2}$ It is possible, although very tedious, to prove this lemma for disconnected floor diagrams $\mathscr{D}$ purely combinatorially. In the interest of a more compact presentation, we chose the route via tropical geometry. ${ }^{3} \mathrm{~A}$ configuration $\left\{\left(x_{i}, y_{i}\right)\right\}$ of $\frac{(d+3) d}{2}-\delta$ points in $\mathbb{R}^{2}$ is horizontally stretched if, for all $i, x_{i}<x_{i+1}$, $y_{i}<y_{i+1}$, and $\min _{i \neq j}\left|x_{i}-x_{j}\right|>\left(d^{3}+d\right) \cdot \max _{i \neq j}\left|y_{i}-y_{j}\right|$.
} 


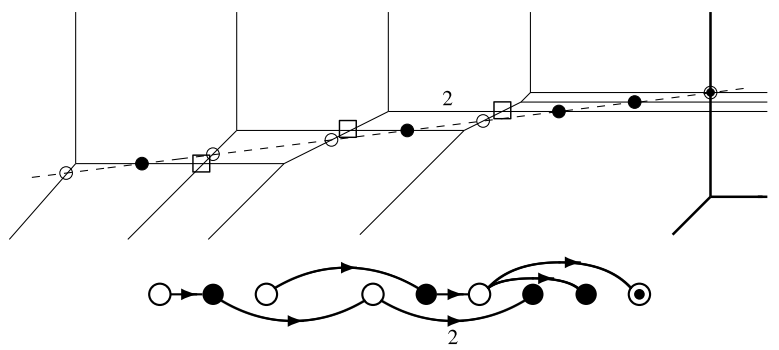

Fig. 3 Correspondence between connected components of a relative marked floor diagram and irreducible components of a tropical plane curve through a horizontally stretched configuration (on a dashed line), tangent to a tropical line (in bold). The three points of intersection of the components of the tropical curves are highlighted by $\square$. The right-most such intersection is of multiplicity $a=2$, the two others of multiplicity $a=1$. Again, we omit writing weights equal to 1

Fig. 4 The intersection of a horizontal weight $a$ edge and a non-horizontal weight 1 edge

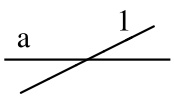

This identification is degree- and genus-preserving, thus $d\left(\mathscr{D}_{j}\right)$ equals the degree ${ }^{4}$ $d\left(C_{i}\right)$ of the tropical curve $C_{i}$ and $\delta\left(\mathscr{D}_{i}\right)$ equals the number $\delta\left(C_{i}\right)$ of tropical nodes of $C_{i}$. As the tropical curve $C$ passes through a horizontally stretched point configuration, all intersection points of $C_{1}$ with $C_{2}$ are locally intersections of a horizontal edge with some weight $a \in \mathbb{Z}_{\geq 1}$ and an edge of slope $\frac{1}{n}$, for $n \in \mathbb{Z}_{\geq 1}$, and weight 1 [6, Sect. 5] (see Fig. 4). Such intersection is of multiplicity $a$ (see, for example, [18, Theorem 4.2]) and thus contributes $a$ to the number of nodes of $C$.

We claim that the number of nodes of $C$ is

$$
\delta(C)=\left(\sum_{j \in J} \delta\left(\Gamma_{j}\right)\right)+\delta(\Lambda, A, B) .
$$

Indeed, the nodes of $C$ come in two types: firstly, bounded horizontal edges $e$ of weight at least 2 contribute $\mathrm{wt}(e)-1$ nodes each $(e$ represents $\operatorname{wt}(e)$ many identified weight- 1 edges, resulting in a genus deficiently of $\mathrm{wt}(e)-1)$. Secondly, intersections of the form as in Fig. 4 contribute $a$ nodes each (cf. with [18, Theorem 4.2]). The former correspond to the edges of $\mathscr{D}$ of weight at least 2 . The latter correspond to pairs $(e, j)$, where $j$ is a vertex of $\mathscr{D}$ and $e$ is either an edge $i \rightarrow k$ of $\mathscr{D}$ of weight $a$, for some $a \in \mathbb{Z}_{\geq 1}$ and $i<j<k$, or an $\alpha$-or $\beta$-edge of the corresponding marking of $\mathscr{D}$ with source $i<j$. Thus, the bounded edges $e$, recorded in $\Gamma_{1}, \ldots, \Gamma_{m}$, and $\Lambda$, contribute $\operatorname{wt}(e) \operatorname{len}(e)-1$ to the number of nodes of $C$. Each of the $a_{i j}$ resp. $b_{i j}$ many $\alpha$-resp. $\beta$-edges of weight $j$ with source $d+1-i$ contribute $j$ nodes. Thus (6) follows by the definition of $\delta\left(\Gamma_{i}\right), \delta(\Lambda), \delta(A)$, and $\delta(B)$.

\footnotetext{
${ }^{4}$ For the purpose of this proof, we only need that there is well defined notion of the degree $d(C)$ of a tropical plane curve $C$, as well as a notion of the number $\delta(C)$ of its nodes (again, see [10, Sects. 2 and 3].
} 
By the tropical Bézout Theorem [18, Theorem 4.2], the tropical curves $C_{1}$ and $C_{2}$ intersect in $d\left(C_{1}\right) d\left(C_{2}\right)=d\left(\mathscr{D}_{1}\right) d\left(\mathscr{D}_{2}\right)$ many nodes. Thus we have

$$
\left(\sum_{j \in J} \delta\left(\Gamma_{j}\right)\right)+\delta(\Lambda, A, B)-d\left(C_{1}\right) \cdot d\left(C_{2}\right)=\delta\left(C_{1}\right)+\delta\left(C_{2}\right)=\delta\left(\mathscr{D}_{1}\right)+\delta\left(\mathscr{D}_{2}\right) .
$$

For floor diagrams $\mathscr{D}$ with two components, the proposition follows from the definition of the cogenus $\delta(\mathscr{D})$. The proof for more than two components is similar.

With an extended template $(\Lambda, A, B)$ we further associate the following numerical data: for $1 \leq j \leq l(\Lambda)$, let $\varkappa_{j}(\Lambda)$ denote the sum of the weights of edges $i \rightarrow k$ of $\Lambda$ with $i<j \leq k$. Define $d_{\min }(\Lambda, A, B)$ to be the smallest positive integer $d$ such that $(\Lambda, A, B)$ can appear (at the right end) in a floor diagram on $\{1,2, \ldots, d\}$. We will see later that $d_{\min }$ is given by an explicit formula. For a matrix $A=\left(a_{i j}\right)$ of non-negative integers with finite support define the "weighted lower sum sequence" wls $(A)$ by

$$
\operatorname{wls}(A)_{i} \stackrel{\text { def }}{=} \sum_{i^{\prime} \geq i, j \geq 1} j \cdot a_{i^{\prime} j}
$$

This sequence records, for each row $i$ of $A$, the sum of entries of $A$ in or below the $i$ th row of $A$, weighted by the column index. As can be seen from Step 2 of Definition 2, wls $(A)_{i}$ resp. wls $(B)_{i}$ equals the total weight of $\alpha$-edges resp. $\beta$-edges that pass vertex $d(\mathscr{D})-i+1$ in an $(\alpha, \beta)$-marking of a floor diagram $\mathscr{D}$ ( $A$ and $B$ are obtained from this marking via (3)).

We now define the number of "markings" of templates and extended templates and relate them to the number of $(\alpha, \beta)$-markings of the corresponding floor diagrams. To each template $\Gamma$, we associate a polynomial: for $k \geq k_{\min }(\Gamma)$, let $\Gamma_{(k)}$ denote the graph obtained from $\Gamma$ by first adding $k+i-1-\varkappa_{i}$ short edges connecting $i-1$ to $i$, for $1 \leq i \leq l(\Gamma)$, and then subdividing each edge of the resulting graph by introducing one new vertex for each edge. The number of short edges in the first step equals the number of edges removed during the template decomposition. For example, if $\Gamma$ is the second template from the top in Fig. 1, then $\Gamma_{(k)}$ has $k-1$ resp. $k$ (subdivided) edges between the first and second resp. second and third vertex of $\Gamma$. By [10, Lemma 5.6] the number of linear extensions (up to equivalence, see the paragraph after Definition 2) of the vertex poset of the graph $\Gamma_{(k)}$ extending the vertex order of $\Gamma$ is given by a polynomial $P_{\Gamma}(k)$ in $k$, whenever $k \geq k_{\min }(\Gamma)$ (see Fig. 1).

For each pair of sequences $(\alpha, \beta)$ and each extended template $(\Lambda, A, B)$ satisfying (5) and $d \geq d_{\min }$, where $d=\sum_{i>1} i\left(\alpha_{i}+\beta_{i}\right)$, we define its "number of markings" as follows. Write $l=l(\Lambda)$ and let $\mathscr{P}(\Lambda, A, B)$ be the poset obtained from $\Lambda$ by

1. first creating an additional vertex $l+1(>l)$,

2. then adding $b_{i j}$ edges of weight $j$ between $l-i$ and $l+1$, for $1 \leq i \leq l$ and $j \geq 1$,

3. then adding $\beta_{j}-\sum_{i \geq 1} b_{i j}$ edges of weight $j$ between $l$ and $l+1$, for $j \geq 1$,

4. then adding

$$
d-l(\Lambda)+i-1-\varkappa_{i}(\Lambda)-\operatorname{wls}(A)_{l+1-i}-\operatorname{wls}(B)_{l+1-i}
$$


("short") edges of weight 1 connecting $i-1$ and $i$, for $1 \leq i \leq l$, and finally

5. subdividing all edges of the resulting graph by introducing a midpoint vertex for each edge.

Example 5 If $(\Lambda, A, B)$ is the extended template of Example 4, then $\mathscr{P}(\Lambda, A, B)$ is (edges are ordered left to right)

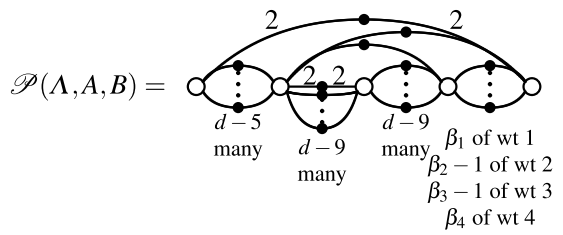

We denote by $Q_{(\Lambda, A, B)}(\alpha ; \beta)$ the number of linear orderings on $\mathscr{P}(\Lambda, A, B)$ (up to equivalence) that extend the linear order on $\Lambda$. As $d \geq d_{\min }(\Lambda, A, B)$ if and only if (8) is non-negative, for $1 \leq i \leq l$, we have

$d_{\min }(\Lambda, A, B)=\max _{1 \leq i \leq l(\Lambda)}\left(l(\Lambda)-i+1+\varkappa_{i}(\Lambda)+\operatorname{wls}(A)_{l(\Lambda)+1-i}+\operatorname{wls}(B)_{l(\Lambda)+1-i}\right)$.

Example 6 Recall from Example 4 that, to construct the floor diagram $\mathscr{D}$ from the extended template $(\Lambda, A, B)$ (and the two templates $\Gamma_{1}$ and $\Gamma_{2}$ ), we had to add (cf. (8)) precisely $d-5, d-9$ and $d-9$ short edges between the last four vertices of $\mathscr{D}$, where $d$ is the degree of $\mathscr{D}$. The invariant $d_{\min }(\Lambda, A, B$, ) measures for which $d$ this is possible. In this example, we have $d_{\min }(\Lambda, A, B)=\max (5,9,9)=9$.

For sequences $s, t_{1}, t_{2}, \ldots$ with $s \geq \sum_{i} t_{i}$ (component-wise), we denote by

$$
\left(\begin{array}{c}
s \\
t_{1}, t_{2}, \ldots
\end{array}\right) \stackrel{\text { def }}{=} \frac{s !}{t_{1} ! t_{2} ! \cdots\left(s-\sum_{i} t_{i}\right) !}
$$

the multinomial coefficient of sequences.

We obtain all $(\alpha, \beta)$-markings of the floor diagram $\mathscr{D}$ that come from a compatible pair of sequences $\left(\left\{\alpha^{i}\right\},\left\{\beta^{i}\right\}\right)$ by independently ordering the $\alpha$-vertices and the non$\alpha$-vertices. The number of such markings is given (via the correspondence (3)) by

$$
\left(\prod_{s=1}^{m} P_{\Gamma_{s}}\left(k_{s}\right)\right) \cdot\left(\begin{array}{c}
\alpha \\
a_{1}^{T}, a_{2}^{T}, \ldots
\end{array}\right) \cdot Q_{(\Lambda, A, B)}(\alpha ; \beta),
$$

where $a_{1}^{T}, a_{2}^{T}, \ldots$ are the column vectors of $A$. We conclude this section by recasting relative Severi degrees in terms of templates and extended templates. 
Proposition 4 For any $\delta \geq 1$, the relative Severi degree $N_{\alpha, \beta}^{\delta}$ is given by

$$
\begin{aligned}
& \sum_{\substack{\left(\Gamma_{1}, \ldots, \Gamma_{m}\right),(\Lambda, A, B)}}\left(\prod_{s=1}^{m} \mu\left(\Gamma_{s}\right) \sum_{k_{1}, \ldots k_{m}} \prod_{s=1}^{m} P_{\Gamma_{s}}\left(k_{s}\right)\right) \\
& \quad \times\left(\mu(\Lambda) \prod_{i \geq 1} i^{\beta_{i}}\left(\begin{array}{c}
\alpha \\
a_{1}, a_{2}, \ldots
\end{array}\right) Q_{(\Lambda, A, B)}(\alpha ; \beta)\right),
\end{aligned}
$$

where the first sum is over all collections $\left(\Gamma_{1}, \ldots, \Gamma_{m}\right)$ of templates and all extended templates $(\Lambda, A, B)$ satisfying $(5), d \geq d_{\min }(\Lambda, A, B)$ and

$$
\sum_{i=1}^{m} \delta\left(\Gamma_{i}\right)+\delta(\Lambda)+\delta(A)+\delta(B)=\delta,
$$

and the second sum is over all positive integers $k_{1}, \ldots, k_{m}$ that satisfy (4).

Proof By Theorem 5, the relative Severi degree is given by

$$
N_{\alpha, \beta}^{\delta}=\sum_{\mathscr{D}} \mu_{\beta}(\mathscr{D}) v_{\alpha, \beta}(\mathscr{D})
$$

with the sum ranging over all floor diagrams $\mathscr{D}$ of degree $d=\sum_{i>1} i\left(\alpha_{i}+\beta_{i}\right)$ and cogenus $\delta$. The result follows from $\mu_{\beta}(\mathscr{D})=\prod_{i \geq 1} i^{\beta_{i}} \cdot\left(\prod_{s=1}^{m} \mu\left(\Gamma_{S}\right)\right) \cdot \mu(\Lambda)$ and (9).

\section{Relative Severi degrees and polynomiality}

We now turn to the proofs of our main results by first showing a number of technical lemmas. For a graph $G$, we denote by $\# E(G)$ the number of edges of $G$. We write $\|A\|_{1}=\sum_{i, j \geq 1}\left|a_{i j}\right|$ for the 1-norm of a (possibly infinite) matrix $A=\left(a_{i j}\right)$.

Lemma 1 For every extended template $(\Lambda, A, B)$, there is a polynomial $q_{(\Lambda, A, B)}$ in $\alpha_{1}, \alpha_{2}, \ldots, \beta_{1}, \beta_{2}, \ldots$ of degree $\# E(\Lambda)+\|B\|_{1}+\delta(B)$ such that, for all $\alpha$ and $\beta$ satisfying (5), the number $Q_{(\Lambda, A, B)}(\alpha ; \beta)$ of linear orderings (up to equivalence) of the poset $\mathscr{P}(\Lambda, A, B)$ is given by

$$
Q_{(\Lambda, A, B)}(\alpha ; \beta)=\frac{(|\beta|-\delta(B)) !}{\beta !} \cdot q_{(\Lambda, A, B)}(\alpha ; \beta)
$$

provided $\sum_{i \geq 1} i\left(\alpha_{i}+\beta_{i}\right) \geq d_{\min }(\Lambda, A, B)$.

Proof We can choose a linear extension of the order on the vertices of $\Lambda$ to the poset $\mathscr{P}(\Lambda, A, B)$ in two steps. First, we choose a linear order on the vertices $0, \ldots, l(\Lambda)+1$, the midpoint vertices of the edges of $\Lambda$, and the midpoint vertices of the edges created in Step 2 in the definition of $\mathscr{P}(\Lambda, A, B)$. In a second step, we 
choose an extension to a linear order on all vertices. There are only finitely many choices in the first step (in particular, they do not involve $\alpha$ and $\beta$ ). Thus, for each choice in the first step, the number of linear extensions in the second step is of the desired form.

Let $r_{i}$ be the number of vertices between $i-1$ and $i$ after the first extension, for $1 \leq i \leq l(\Lambda)+1$, and let $\sigma_{i}$ be the number of equivalent such linear orderings of the interval between $i-1$ and $i$ ( $\sigma_{i}$ is independent of the particular choice of the linear order). To insert the additional vertices (up to equivalence) between the vertices 0 and $l=l(\Lambda)$ we have

$$
\prod_{i=1}^{l} \frac{1}{\sigma_{i}}\left(\begin{array}{c}
d-l(\Lambda)+i-1-\varkappa_{i}(\Lambda)-\operatorname{wls}(A)_{l+1-i}-\operatorname{wls}(B)_{l+1-i}+r_{i} \\
r_{i}
\end{array}\right)
$$

many possibilities where again $d=\sum_{i \geq 1} i\left(\alpha_{i}+\beta_{i}\right)$. If $d \geq d_{\min }(\Lambda, A, B)$ then expression (11) is a polynomial in $d$ of degree $\sum_{i=1}^{l} r_{i}$, and thus in $\alpha_{1}, \alpha_{2}, \ldots, \beta_{1}$, $\beta_{2}, \ldots$ The number of (equivalent) orderings of the vertices between $l$ and $l+1$ is the multinomial coefficient

$$
\left(\begin{array}{c}
|\beta|-\|B\|_{1}+r_{l+1} \\
\beta_{1}-\left|b_{1}^{T}\right|, \beta_{2}-\left|b_{2}^{T}\right|, \ldots
\end{array}\right)
$$

where $\left|b_{j}^{T}\right|$ denotes the sum of the entries in the $j$ th column of $B$. As $\|B\|_{1} \leq \delta(B)$, expression (12) equals, for all $\beta_{1}, \beta_{2}, \ldots \geq 0$,

$$
\left(\begin{array}{c}
|\beta| \\
\beta_{1}, \beta_{2}, \ldots
\end{array}\right) \frac{(|\beta|-\delta(B)) !}{|\beta| !} P(\beta)=\frac{(|\beta|-\delta(B)) !}{\beta !} P(\beta)
$$

for a polynomial $P$ in $\beta_{1}, \beta_{2}, \ldots$ of degree $r_{l+1}+\delta(B)$. The product of (11) and (13) is

$$
\frac{(|\beta|-\delta(B)) !}{\beta !} P^{\prime}(\alpha ; \beta)
$$

for a polynomial $P^{\prime}$ in $\alpha_{1}, \alpha_{2}, \ldots, \beta_{1}, \beta_{2}, \ldots$ of degree $\# E(\Lambda)+\|B\|_{1}+\delta(B)$, provided $d \geq d_{\min }(\Lambda, A, B)$, where we used that $\sum_{i=1}^{l+1} r_{i}=\# E(\Lambda)+\|B\|_{1}$. As (14) equals the number of linear extensions (up to equivalence) that can be obtained by linearly ordering the vertices in all segments between $i-1$ and $i$, for $1 \leq i \leq l+1$, the proof is complete.

In Sect. 3, we defined, for an extended template $(\Lambda, A, B)$, the invariant

$$
d_{\min }=\max _{1 \leq i \leq l(\Lambda)}\left(l(\Lambda)-i+1+\varkappa_{i}(\Lambda)+\operatorname{wls}(A)_{l(\Lambda)+1-i}+\operatorname{wls}(B)_{l(\Lambda)+1-i}\right) .
$$

It equals the minimal $d \geq 1$ so that $(\Lambda, A, B)$ can appear in the relative decomposition of a floor diagram of degree $d$. Let $i_{0}$ be the smallest $i$ for which the maximum in (15) is attained. Define the quantity $s(\Lambda, A, B)$ to be the number of edges of $\Lambda$ 
from $i_{0}-1$ to $i_{0}$ (of any weight). For example, if $(\Lambda, A, B)$ is the extended template

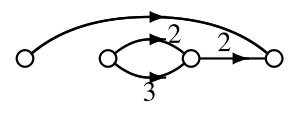

$$
A=\left[\begin{array}{cccc}
1 & 0 & 0 & \ldots \\
1 & 0 & 0 & \ldots \\
0 & 0 & 0 & \ldots \\
\vdots & \vdots & \vdots & \ddots
\end{array}\right]
$$$$
B=\left[\begin{array}{cccc}
0 & 1 & 0 & \cdots \\
0 & 0 & 0 & \cdots \\
0 & 0 & 0 & \cdots \\
\vdots & \vdots & \vdots & \ddots
\end{array}\right]
$$

then tracing through the definition yields $d_{\min }=\max (4,11,8)=11$. The maximum is attained at $i_{0}=2$, and there are $s=2$ edges in $\Lambda$ between vertices 1 and 2 .

Lemma 2 For any extended template $(\Lambda, A, B)$ and any $\alpha, \beta \geq 0$ (component-wise) with

$$
d_{\min }(\Lambda, A, B)-s(\Lambda, A, B) \leq \sum_{i \geq 1} i\left(\alpha_{i}+\beta_{i}\right) \leq d_{\min }(\Lambda, A, B)-1,
$$

we have $q_{(\Lambda, A . B)}(\alpha ; \beta)=0$, where $q_{(\Lambda, A, B)}$ is the polynomial of Lemma 1 .

Proof Notice that $d_{\min }-l(\Lambda)+i_{0}-1=\varkappa_{i_{0}}(\Lambda)+\operatorname{wls}(A)_{l(\Lambda)+1-i_{0}}+$ wls $(B)_{l(\Lambda)+1-i_{0}}$, where $d_{\min }=d_{\min }(\Lambda, A, B)$. Therefore, the number of short edges added between $i_{0}-1$ and $i_{0}$ in Step 3 of the definition of the poset $\mathscr{P}(\Lambda, A, B)$ is $d-d_{\min }$, where as before $d=\sum_{i>1} i\left(\alpha_{i}+\beta_{i}\right)$. Recall that, up to the factor $\frac{(|\beta|-\delta(B)) !}{\beta !}$, the polynomial $q_{(\Lambda, A, B)}$ records the number of linear extensions of the poset $\mathscr{P}(\Lambda, A, B)$ (up to equivalence). Every such extension is obtained by, first, linearly ordering the $d-d_{\min }$ midpoints of the short edges between $i_{0}-1$ and $i_{0}$ that were added in Step 3 together with the $s(\Lambda, A, B)$ midpoints of the edges of $\Lambda$ between $i_{0}-1$ and $i_{0}$, before extending this to a linear order on all the vertices of $\mathscr{P}(\Lambda, A, B)$. Let $r_{i_{0}}$ be the number of midpoint vertices between $i_{0}-1$ and $i_{0}$ after a particular first extension. Then $\left(\begin{array}{c}d-d_{\min }+r_{i} \\ r_{i_{0}}\end{array}\right)$ is a factor of the polynomial counting the linear extensions on all vertices. As $r_{i_{0}} \geq s$ for each choice of first extension, $q_{(\Lambda, A, B)}$ is divisible by the polynomial $\left(d-d_{\min }+1\right) \cdots\left(d-d_{\min }+s(\Lambda, A, B)\right)$.

The next lemma specifies which extended templates are compatible with a given degree.

Lemma 3 For every extended template $(\Lambda, A, B)$ we have

$$
d_{\min }(\Lambda, A, B)-s(\Lambda, A, B) \leq \delta(\Lambda)+\delta(A)+\delta(B)+1 .
$$

Proof We use the notation from above and write $l=l(\Lambda)$. Notice that

$$
d_{\min }(\Lambda, A, B)-l(\Lambda)+i_{0}-1=\varkappa_{i_{0}}(\Lambda)+\operatorname{wls}(A)_{l+1-i_{0}}+\operatorname{wls}(B)_{l+1-i_{0}} .
$$

Therefore, it suffices to show

$$
\begin{aligned}
l(\Lambda) \leq & \delta(\Lambda)-\varkappa_{i_{0}}(\Lambda)+s(\Lambda, A, B)+\delta(A)-\operatorname{wls}(A)_{l+1-i_{0}} \\
& +\delta(B)-\operatorname{wls}(B)_{l+1-i_{0}}+i_{0} .
\end{aligned}
$$


Let $\Lambda^{\prime}$ be the graph obtained from $\Lambda$ by, firstly, removing all edges $j \rightarrow k$ with either $k<i_{0}$ or $j \geq i_{0}$ and, secondly, deleting all vertices $j$ for which there is no edge $i \rightarrow k$ in the new graph with $i \leq j \leq k$. It is easy to see that $l(\Lambda, A, B)-l\left(\Lambda^{\prime}, A, B\right) \leq$ $\delta(\Lambda)-\delta\left(\Lambda^{\prime}\right)$. Thus, we can assume without loss of generality that all edges $j \rightarrow k$ of $\Lambda$ satisfy $j<i_{0} \leq k$. Therefore, as $\varkappa_{i_{0}}(\Lambda)=\sum$ edges $e$ wt $(e)$, we have

$$
\begin{aligned}
\delta(\Lambda)-\varkappa_{i_{0}}+s(\Lambda, A, B) & =\sum_{\text {edges } e}(\operatorname{wt}(e)(\operatorname{len}(e)-1)-1)+s \\
& =\sum_{e: \operatorname{len}(e) \geq 2}(\operatorname{wt}(e)(\operatorname{len}(e)-1)-1),
\end{aligned}
$$

where, again, len $(e)$ is the length $k-j$ of an edge $j \stackrel{e}{\rightarrow} k$. Thus, we need to show that

$$
\begin{aligned}
l(\Lambda) \leq & \sum_{e: \operatorname{len}(e) \geq 2}(\operatorname{wt}(e)(\operatorname{len}(e)-1)-1) \\
& +\delta(A)-\operatorname{wls}(A)_{l+1-i_{0}}+\delta(B)-\operatorname{wls}(B)_{l+1-i_{0}}+i_{0} .
\end{aligned}
$$

It is easy to see that the matrix $A$ satisfies $\delta(A) \geq \operatorname{wls}(A)_{i}+l(A)-1$ for all $i \geq 1$, therefore, if $l(A)=l(\Lambda)$, it suffices to show that

$$
l(A) \leq \sum_{e: \operatorname{len}(e) \geq 2}(\operatorname{wt}(e)(\operatorname{len}(e)-1)-1)+l(A)-1+\delta(B)-\operatorname{wls}(B)_{l+1-i_{0}}+i_{0} .
$$

But (17) is clear as all summands in the sum over the edges of $\Lambda$ are non-negative and $\delta(B) \geq \operatorname{wls}(B)_{l+1-i_{0}}$. The same argument also settles the case $l(B)=l(\Lambda)$.

Otherwise, we can assume that $l(\Lambda)>l(A) \geq l(B)$ and that there exists an edge $0 \rightarrow i$ of $\Lambda$ with $l(\Lambda)-l(A) \leq i-1$. Assume, additionally, that $i_{0} \leq l(\Lambda)-l(A)$ which implies wls $(A)_{l+1-i_{0}}=0$.Then we have $l(\Lambda) \leq i-1+l(A)$, and, again using $\delta(B) \geq \operatorname{wls}(B)_{l+1-i_{0}}$, it suffices to show that

$$
i-1+l(A) \leq \sum_{e: \operatorname{len}(e) \geq 2}(\operatorname{wt}(e)(\operatorname{len}(e)-1)-1)+\delta(A)+i_{0} .
$$

But this inequality is clear as

$$
\sum_{e: \operatorname{len}(e) \geq 2}(\operatorname{wt}(e)(\operatorname{len}(e)-1)-1) \geq i-2,
$$

together with $l(A) \leq \delta(A)$ and $i_{0} \geq 1$.

Finally, it remains to show that $l(\Lambda)>l(A) \geq l(B)$ and $i_{0} \geq l(\Lambda)-l(A)+1$ imply (16). Using $\sum(\operatorname{wt}(e)(\operatorname{len}(e)-1)-1) \geq 0$ and $\delta(B) \geq \operatorname{wls}(B)_{l+1-i_{0}}$, it suffices to show

$$
l(\Lambda) \leq \delta(A)-\operatorname{wls}(A)_{l+1-i_{0}}+i_{0} .
$$

We have (by definition of $\delta(A)$ and wls $(A)_{l+1-i_{0}}$ ) that

$$
\delta(A)-\operatorname{wls}(A)_{l+1-i_{0}}+i_{0}=\sum(i-1) j a_{i j}+\sum i j a_{i j}+i_{0},
$$


where the first sum runs over $i \geq l+1-i_{0}, j \geq 1$ and the second sum runs over $1 \leq i<l+1-i_{0}, j \geq 1$. As $i_{0} \geq l(\Lambda)-l(A)+1$ there exists a non-zero entry $a_{i^{\prime} j^{\prime}}$ of $A$ with $i^{\prime}=l(A) \geq l+1-i_{0}$. Therefore, the index set of the first sum of (19) is non-empty and the right-hand side of (19) is greater or equal to $i^{\prime}-1+i_{0}=l(\Lambda)$ as $i_{0} \geq l(\Lambda)-l(A)+1$. This shows (18) and completes the proof.

Before we turn to the proof of the main theorem of this paper, we introduce a last numerical invariant $s(\Gamma)$ associated with each template $\Gamma$. The definition of $s(\Gamma)$ parallels that of $s(\Lambda, A, B)$ for extended templates $(\Lambda, A, B)$. This invariant is necessary to establish the polynomiality threshold of Theorem 1.

Recall from Sect. 3 that, for a template $\Gamma$, we defined

$$
k_{\min }(\Gamma)=\max _{1 \leq j \leq l(\Gamma)}\left(\varkappa_{j}(\Gamma)-i+1\right)
$$

where $\varkappa_{j}(\Gamma)$ is the total weight of all edges $i \rightarrow k$ of $\Gamma$ with $i<j \leq k$. Let $j_{0}(\Gamma)$ be the smallest $j$ for which the maximum is attained and define $s(\Gamma)$ to be the number of edges of $\Gamma$ from $j_{0}-1$ to $j_{0}$. See Fig. 1 for examples. Then one can show the following; the proof is along similar lines as for our Lemma 3.

Lemma 4 [3, Lemma 4.3] Each template $\Gamma$ satisfies

$$
k_{\min }(\Gamma)+l(\Gamma)-s(\Gamma) \leq \delta(\Gamma)+1 .
$$

Proof of Theorem 1 We first show that (1) holds of all $\alpha, \beta$ with $d \geq \delta+1$, where we again write $d=\sum_{i>1} i\left(\alpha_{i}+\beta_{i}\right)$. This implies (1) for all $\alpha$ and $\beta$, for which at least one of $\alpha_{1}, \alpha_{2}, \ldots, \beta_{2}, \beta_{3}, \ldots$ is non-zero (note that $\beta_{1}$ is omitted): in that case $|\beta| \geq \delta$ implies $d \geq \delta+1$.

Notice that we can remove condition (5) from formula (10) of Proposition 4 and still obtain correct relative Severi degrees as $\left(\begin{array}{c}\alpha \\ a_{1}^{T}, a_{2}^{T}, \ldots\end{array}\right) Q_{(\Lambda, A, B)}(\alpha ; \beta)=0$ whenever (5) is violated. The first factor of (10) equals

$$
\sum_{k_{m}=k_{\min }\left(\Gamma_{m}\right)}^{d-l(\Lambda)} \mu\left(\Gamma_{m}\right) P_{\Gamma_{m}}\left(k_{m}\right) \sum_{k_{m-1}=k_{\min }\left(\Gamma_{m-1}\right)}^{k_{m}-l\left(\Gamma_{m-1}\right)} \cdots \sum_{k_{1}=k_{\min }\left(\Gamma_{1}\right)}^{k_{2}-l\left(\Gamma_{1}\right)} \mu\left(\Gamma_{1}\right) P_{\Gamma_{1}}\left(k_{1}\right)
$$

and is, therefore, an iterated "discrete integral" of polynomials. Expression (20) is polynomial in $d$, provided $d$ is large enough (as discrete integration preserves polynomiality, see e.g. Faulhaber's formula [3, Lemma 3.5]). Furthermore, as the polynomials $P_{\Gamma_{i}}\left(k_{i}\right)$ have degrees \#E $\left(\Gamma_{i}\right)$ and each "discrete integration" increases the degree by 1 , the polynomial (20) (if $d$ is large enough) is of degree $\sum_{i=1}^{m} \# E\left(\Gamma_{i}\right)+m$.

We claim that (20) is a polynomial in $d$ provided that $d-l(\Lambda) \geq \sum_{i=1}^{m} \delta\left(\Gamma_{i}\right)+1$. (Readers interested solely in polynomiality of relative Severi degrees for large enough $\alpha$ and $\beta$ may skip this paragraph. Also, computational evidence suggests that this bound, without the " +1 " is sharp in general). Indeed, by [3, Lemma 3.6] and repeated application of [3, Lemma 4.1] and [3, Lemma 4.2], it suffices to show that $d-l(\Lambda) \geq$ 
$\sum_{i=1}^{m} \delta\left(\Gamma_{i}\right)+1$ simultaneously implies

$$
\begin{aligned}
& d \geq l\left(\Gamma_{m}\right)+k_{\min }\left(\Gamma_{m}\right)-s\left(\Gamma_{m}\right)-1, \\
& d \geq l\left(\Gamma_{m}\right)+l\left(\Gamma_{m-1}\right)+k_{\min }\left(\Gamma_{m-1}\right)-s\left(\Gamma_{m-1}\right)-2, \\
& \quad \vdots \\
& d \geq l\left(\Gamma_{m}\right)+l\left(\Gamma_{m-1}\right)+\cdots+l\left(\Gamma_{1}\right)+k_{\min }\left(\Gamma_{1}\right)-s\left(\Gamma_{1}\right)-m,
\end{aligned}
$$

for all collections of templates $\left(\Gamma_{1}, \ldots, \Gamma_{m}\right)$ with $\sum_{i=1}^{m} \delta\left(\Gamma_{i}\right)=\delta$. The first inequality follows directly from Lemma 4 . For the other inequalities, notice that $l\left(\Gamma_{i}\right)-1 \leq \delta\left(\Gamma_{i}\right)$ for all templates $\Gamma_{i}$ in the collection. Fix $1 \leq i_{0} \leq m$, then Lemma 4 applied to $\Gamma_{i_{0}}$ says

$$
l\left(\Gamma_{i_{0}}\right)+k_{\min }\left(\Gamma_{i_{0}}\right)-s\left(\Gamma_{i_{0}}\right) \leq \delta\left(\Gamma_{i_{0}}\right)+1 .
$$

Thus the right-hand-side of the $\left(m-i_{0}+1\right)$ th inequality of $(21)$ is less than or equal to

$$
\sum_{i=i_{0}}^{m} \delta\left(\Gamma_{i}\right)+1 \leq d-l(\Lambda) \leq d .
$$

Furthermore, we have $l(\Lambda) \leq \delta(\Lambda)+\delta(A)+\delta(B)$. (By the "connectedness" property of extended templates, each vertex $1 \leq j \leq l(\Lambda)-\max (l(A), l(B))$ of $\Lambda$ is passed by an edge of $\Lambda$. Therefore, we have $\delta(\Lambda)) \geq l(\Lambda)-\max (l(A), l(B))$. Notice that $l(A) \leq \delta(A)$ and $l(B) \leq \delta(B)$.) Therefore, the first factor of (10) is a polynomial in $d$ if $d \geq \delta+1=\left(\sum_{i} \delta\left(\Gamma_{i}\right)\right)+1+\delta(\Lambda)+\delta(A)+\delta(B)$ (again computation evidence suggests that this bound, without the " +1 ", is sharp).

For column vectors $a_{1}^{T}, a_{2}^{T}, \ldots$ of a matrix $A$, the multinomial coefficient $\left(\begin{array}{c}\alpha \\ a_{1}^{T}, a_{2}^{T}, \ldots\end{array}\right)$ is a polynomial of degree $\|A\|_{1}$ in $\alpha_{1}, \alpha_{2}, \ldots$, if $\alpha_{1}, \alpha_{2}, \ldots \geq 0$. For an extended template $(\Lambda, A, B)$, define

$$
R_{(\Lambda, A, B)}(\alpha ; \beta) \stackrel{\text { def }}{=}\left(\begin{array}{c}
\alpha \\
a_{1}^{T}, a_{2}^{T}, \ldots
\end{array}\right) \cdot \frac{(|\beta|-\delta(B)) !}{(|\beta|-\delta) !} \cdot q_{(\Lambda, A, B)}(\alpha ; \beta),
$$

where $q_{(\Lambda, A, B)}(\alpha ; \beta)$ is the polynomial of Lemma 1 . By Lemma 1 and as $\delta(B) \leq \delta$, $R_{(\Lambda, A, B)}(\alpha ; \beta)$ is a polynomial in $\alpha$ and $\beta$ of degree $\# E(\Lambda)+\|A\|_{1}+\|B\|_{1}+\delta$, provided $d \geq d_{\min }(\Lambda, A, B)$. The second factor of (10) then equals

$$
\prod_{i \geq 1} i^{\beta_{i}} \cdot \frac{(|\beta|-\delta) !}{\beta !} \cdot R_{(\Lambda, A, B)}(\alpha ; \beta) .
$$

By Lemma 2, the second factor of (10) equals expression (22) for all $\alpha, \beta$ with $d \geq$ $d_{\text {min }}(\Lambda, A, B)-s(\Lambda, A, B)$. Thus, using Lemma 3 , if

$$
d \geq \delta+1 \geq \delta(\Lambda)+\delta(A)+\delta(B)+1 \geq d_{\min }(\Lambda, A, B)-s(\Lambda, A, B)
$$

the second factor in (10) is $\prod_{i \geq 1} i^{\beta_{i}} \cdot \frac{(|\beta|-\delta) !}{\beta !}$ times a polynomial in $\alpha_{1}, \alpha_{2}, \ldots, \beta_{1}$, $\beta_{2}, \ldots$ of degree $\# E(\Lambda)+\|A\|_{1}+\|B\|_{1}+\delta$. Hence (1) holds if $|\beta| \geq \delta$ and at least 
one $\beta_{i}$, for $i \geq 2$, or one $\alpha_{i}$, for $i \geq 1$, is non-zero. Notice that each summand of (10) contributes a polynomial of degree

$$
\sum_{i=1}^{m} \# E\left(\Gamma_{i}\right)+m+\# E(\Lambda)+\|A\|_{1}+\|B\|_{1}+\delta
$$

to the relative node polynomial $N_{\delta}(\alpha ; \beta)$. It is not hard to see that expression (23) is at most $3 \delta$, and that equality is attained by letting $\Gamma_{1}, \ldots, \Gamma_{\delta}$ be the unique template on three vertices with cogenus 1 (see Fig. 1$)$ and $(\Lambda, A, B)$ be the unique extended template of cogenus 0 (see Fig. 2).

If $\alpha=0$ and $\beta=(d, 0, \ldots)$, then $N_{\alpha, \beta}^{\delta}$ equals the (non-relative) Severi degree $N^{d, \delta}$, which, in turn, is given by the (non-relative) node polynomial $N_{\delta}^{\mathrm{nr}}(d)$ provided $d \geq \delta$ (see [3, Theorem 1.3]). Therefore, we have $N_{\delta}(0 ; d)=N_{\delta}^{\mathrm{nr}}(d)$. $d(d-1) \cdots(d-\delta+1)$ as polynomials in $d$. Applying [3, Theorem 1.3] again finishes the proof.

Remark 1 Expression (10) gives, in principle, an algorithm to compute the relative node polynomial $N_{\delta}(\alpha ; \beta)$, for any $\delta \geq 1$. In [3, Sect. 3] we explain how to generate all templates of a given cogenus, and how to compute the first factor in (10). The generation of all extended templates of a given cogenus from the templates is straightforward, as is the computation of the second factor in (10).

Remark 2 The proof of Theorem 1 simplifies if we relax the polynomiality threshold. More specifically, without considering the quantity $s(\Lambda, A, B)$ and the rather technical Lemmas 2 and 3, the argument still implies (1) provided $|\beta| \geq 2 \delta$ (instead of $|\beta| \geq \delta)$.

The conclusion from the proof of Theorem 1 is two-fold.

Proof of Proposition 1 Every extended template $(\Lambda, A, B)$ considered in (10) satisfies $\delta(A) \leq \delta$ and $\delta(B) \leq \delta$. Therefore, all rows $i>\delta$ in $A$ or $B$ are zero.

Proof of Theorem 2 By the proof of Lemma 1 we have, for every extended template $(\Lambda, A, B)$,

$$
R_{(\Lambda, A, B)}(\alpha, 0 ; \beta)=R_{(\Lambda, A, B)}(\alpha ; \beta), \quad R_{(\Lambda, A, B)}(\alpha ; \beta, 0)=R_{(\Lambda, A, B)}(\alpha ; \beta) .
$$

Hence, by the proof of Theorem 1 , the result follows.

Now it is also easy to prove Theorem 3.

Proof of Theorem 3 Proposition 4 gives a combinatorial description of relative Severi degrees. The proof of Lemma 1 provides a method to calculate the polynomial $Q_{(\Lambda, A, B)}(\alpha ; \beta)$. All terms of expression (10) are explicit or can be evaluated using the techniques of $[3$, Sect. 3]. This reduces the calculation to a (non-trivial) computer calculation. 


\section{Coefficients of relative node polynomials}

We now turn toward the computation of the coefficients of the relative node polynomial $N_{\delta}(\alpha ; \beta)$ of large degree for any $\delta$. By Theorem 1 , the polynomial $N_{\delta}(\alpha, \beta)$ is of degree $3 \delta$. In the following we propose a method to compute all terms of $N_{\delta}(\alpha ; \beta)$ of degree $\geq 3 \delta-t$, for any given $t \geq 0$. This method was used (with $t=2$ ) to compute the terms in Theorem 4.

The main idea of the algorithm is that, even for general $\delta$, only a small number of summands of (10) contribute to the terms of $N_{\delta}(\alpha ; \beta)$ of large degree. A summand of (10) is indexed by a collection of templates $\tilde{\Gamma}=\left\{\Gamma_{s}\right\}$ and an extended template $(\Lambda, A, B)$. To determine whether this summand actually contributes to $N_{\delta}(\alpha ; \beta)$ we define the (degree) defects

- of the collection of templates $\tilde{\Gamma}$ by

$$
\operatorname{def}(\tilde{\Gamma}) \stackrel{\operatorname{def}}{=}\left(\sum_{s=1}^{m} \delta\left(\Gamma_{i}\right)\right)-m, \quad \text { and }
$$

- of the extended template $(\Lambda, A, B)$ by

$$
\operatorname{def}(\Lambda, A, B) \stackrel{\text { def }}{=} \delta(\Lambda)+2 \delta(A)+2 \delta(B)-\|A\|_{1}-\|B\|_{1}
$$

The following lemma restricts the indexing set of (10) to the relevant terms, if only the leading terms of $N_{\delta}(\alpha ; \beta)$ are of interest.

Lemma 5 The summand of (10) indexed by $\tilde{\Gamma}$ and $(\Lambda, A, B)$ is of the form

$$
1^{\beta_{1}} 2^{\beta_{2}} \ldots \frac{(|\beta|-\delta) !}{\beta !} \cdot P(\alpha ; \beta),
$$

where $P(\alpha ; \beta)$ is a polynomial in $\alpha_{1}, \alpha_{2}, \ldots, \beta_{1}, \beta_{2}, \ldots$ of degree $\leq 3 \delta-\operatorname{def}(\tilde{\Gamma})-$ $\operatorname{def}(\Lambda, A, B)$.

Proof By [3, Lemma 5.2], the first factor of (10) is of degree at most

$$
2 \cdot \sum_{s=1}^{m} \delta\left(\Gamma_{s}\right)-\sum_{s=1}^{m}\left(\delta\left(\Gamma_{s}\right)-1\right)=\sum_{s=1}^{m} \delta\left(\Gamma_{S}\right)+m
$$

The multinomial coefficient $\left(\begin{array}{c}\alpha \\ a_{1}^{T}, a_{2}^{T}, \ldots\end{array}\right)$ is a polynomial in $\alpha$ of degree $\|A\|_{1}$ if $a_{j}^{T}$ are the $j$ th column vector of the matrix $A$. Recall from the proof of Theorem 1 that the second factor of (10) is

$$
\prod_{i \geq 1} i^{\beta_{i}} \frac{(|\beta|-\delta) !}{\beta !} \text { times a polynomial in } \alpha, \beta \text { of degree } \# E(\Lambda)+\|A\|_{1}+\|B\|_{1}+\delta .
$$


Therefore, the contribution of this summand to the relative node polynomial is of degree at most

$$
\begin{aligned}
& \sum_{s=1}^{m} \delta\left(\Gamma_{s}\right)+m+\# E(\Lambda)+\|A\|_{1}+\|B\|_{1}+\delta \\
& \quad=3 \delta-2 \sum_{s=1}^{m} \delta\left(\Gamma_{s}\right)-2 \delta(\Lambda)-2 \delta(A)-2 \delta(B)+\# E(\Lambda) \\
& \quad=3 \delta-\operatorname{def}(\tilde{\Gamma})-\operatorname{def}(\Lambda, A, B)-\delta(\Lambda)+\# E(\Lambda)
\end{aligned}
$$

The result follows as $\delta(\Lambda) \geq \# E(\Lambda)$.

Therefore, to compute the coefficients of degree $\geq 3 \delta-t$ of $N_{\delta}(\alpha ; \beta)$ for some $t \geq 0$, it suffices to consider only summands of (10) with $\operatorname{def}(\tilde{\Gamma}) \leq t$ and $\operatorname{def}(\Lambda, A, B) \leq t$.

One can proceed as follows. First, we can compute, for some formal variable $\tilde{\delta}$, the terms of degree $\geq 2 \tilde{\delta}-t$ of the first factor of (10) to $N_{\tilde{\delta}}(\alpha ; \beta)$, that is, the terms of degree $\geq 2 \tilde{\delta}-t$ of

$$
R_{\tilde{\delta}}(d) \stackrel{\text { def }}{=} \sum \prod_{i=1}^{m} \mu\left(\Gamma_{i}\right) \sum_{k_{m}=k_{\min }\left(\Gamma_{m}\right)}^{d-l\left(\Gamma_{m}\right)} P_{\Gamma_{m}}\left(k_{m}\right) \cdots \sum_{k_{1}=k_{\min }\left(\Gamma_{1}\right)}^{k_{2}-l\left(\Gamma_{1}\right)} P_{\Gamma_{1}}\left(k_{1}\right),
$$

where the first sum is over all collections of templates $\tilde{\Gamma}=\left(\Gamma_{1}, \ldots, \Gamma_{m}\right)$ with $\delta(\tilde{\Gamma})=\tilde{\delta}$. (Notice that (24) is expression [10, (5.13)] without the " $\varepsilon$-correction" in the sum indexed by $k_{m}$.) The leading terms of $R_{\tilde{\delta}}(d)$ can be computed with a slight modification of [3, Algorithm 2] (by replacing, in the notation of [3], $C^{\text {end }}$ by $C$ and $M^{\text {end }}$ by $M$ ). The algorithm relies on the polynomiality of solutions of certain polynomial difference equations, which has been verified for $t \leq 7$, see [3, Sect. 5] for more details. With a Maple implementation of this algorithm one obtains (with $t=5)$

$$
\begin{aligned}
R_{\tilde{\delta}}(d)= & \frac{3^{\tilde{\delta}}}{\tilde{\delta} !}\left[d^{2 \tilde{\delta}}-\frac{8 \tilde{\delta}}{3} d^{2 \tilde{\delta}-1}+\frac{\tilde{\delta}(11 \tilde{\delta}+1)}{3^{2}} d^{2 \tilde{\delta}-2}+\frac{\tilde{\delta}(\tilde{\delta}-1)(496 \tilde{\delta}-245)}{6 \cdot 3^{3}} d^{2 \tilde{\delta}-3}\right. \\
& -\frac{\tilde{\delta}(\tilde{\delta}-1)\left(1685 \tilde{\delta}^{2}-2773 \tilde{\delta}+1398\right)}{6 \cdot 3^{4}} d^{2 \tilde{\delta}-4} \\
& \left.-\frac{\tilde{\delta}(\tilde{\delta}-1)(\tilde{\delta}-2)\left(7352 \tilde{\delta}^{2}+11611 \tilde{\delta}-25221\right)}{30 \cdot 3^{5}} d^{2 \tilde{\delta}-5}+\cdots\right] .
\end{aligned}
$$

Finally, to compute the coefficients of degree $\geq 3 \delta-t$, it remains to compute all extended templates $(\Lambda, A, B)$ with $\operatorname{def}(\Lambda, A, B) \leq t$ and collect the terms of degree $\geq 3 \delta-t$ of the polynomial

$$
R_{\tilde{\delta}}(d-l(\Lambda)) \cdot \mu(\Lambda)\left(\begin{array}{c}
\alpha \\
a_{1}^{T}, a_{2}^{T}, \ldots
\end{array}\right) \prod_{i=\delta(B)}^{\delta-1}(|\beta|-i) \cdot q_{(\Lambda, A, B)}(\alpha ; \beta),
$$


where, as before, $a_{1}^{T}, a_{2}^{T}, \ldots$ denote the column vectors of the matrix $A, q_{(\Lambda, A, B)} \times$ $(\alpha ; \beta)$ is the polynomial of Lemma 1 , and $\tilde{\delta}=\delta-\delta(\Lambda, A, B)$. Notice that, for an indeterminant $x$ and integers $c \geq 0$ and $\delta \geq 1$, we have the expansion

$$
\prod_{i=c}^{\delta-1}(x-i)=\sum_{t=0}^{\delta-c} s(\delta-c, \delta-c-t)(x-c)^{\delta-c-t},
$$

where $s(n, m)$ is the Stirling number of the first kind [21, Sect. 1.3] for integers $n, m \geq 0$. Furthermore, with $\delta^{\prime}=\delta-c$ the coefficients $s\left(\delta^{\prime}, \delta^{\prime}-t\right)$ of the right-handside of (26) equal $\delta^{\prime}\left(\delta^{\prime}-1\right) \cdots\left(\delta^{\prime}-t\right) \cdot S_{t}\left(\delta^{\prime}\right)$, where $S_{t}$ is the $t$ th Stirling polynomial [13, (6.45)], for $t \geq 0$, and thus are polynomial in $\delta^{\prime}$. Therefore, we can compute the leading terms of the product in (25) by collecting the leading terms in the sum expansion above.

Proof of Proposition 2 Using [3, Algorithm 2] we can compute the terms of the polynomial $R_{\tilde{\Gamma}}(d)$ of degree $\geq 2 \tilde{\delta}-7$ (see [3, Sect. 5]) and observe that all coefficients are polynomial in $\tilde{\delta}$. By the previous paragraph, the coefficients of the expansion of the sum of (25) are polynomial in $\delta$. This completes the proof.

Proof of Theorem 4 The method described above is a direct implementation of formula (10), which equals the relative Severi degree by the proof of Theorem 1.

Remark 3 It is straightforward to compute the coefficients of $N_{\delta}(\alpha ; \beta)$ of degree $\geq 3 \delta-7$ (and thereby to extend Theorem 4). In particular, one can see that the terms $\alpha_{2}$ and $\beta_{2}$ (by themselves) appear in $N_{\delta}(\alpha ; \beta)$ in degree $3 \delta-3$. Algorithm 3 of [3] computes the coefficients of the polynomials $R_{\tilde{\delta}}(d)$ of degree $\geq 2 \tilde{\delta}-7$, and thus the desired terms can be collected from (25). We expect this method to compute the leading terms of $N_{\delta}(\alpha, \beta)$ of degree $\geq 3 \delta-t$ for arbitrary $t \geq 0$ (see [3, Sect. 5], especially Conjecture 5.5).

Acknowledgements The author thanks the referees for helpful and careful comments and suggestion that led to significant improvements of the article. The author was partially supported by a Rackham OneTerm Dissertation Fellowship and by the NSF grant DMS-055588.

\section{Appendix: the first three relative node polynomials}

Below we list the relative node polynomials $N_{\delta}(\alpha ; \beta)$ for $\delta \leq 3$. For $\delta \leq 6$, the polynomials $N_{\delta}(\alpha ; \beta)$ are as provided in the ancillary files of the arXiv-version of this paper [2]. All polynomials were obtained by a Maple implementation of the formula (10). See Remark 1 for more details. For $\delta \leq 1$ this agrees with [10, Corollary 4.5, 4.6]. As before, we write $d=\sum_{i>1} i\left(\alpha_{i}+\beta_{i}\right)$. By Theorem 1 the relative Severi degrees $N_{\alpha, \beta}^{\delta}$ are given by $N_{\alpha, \beta}^{\delta}=1^{\beta_{1}} 2^{\beta_{2}} \ldots \frac{(|\beta|-\delta) !}{\beta !} N_{\delta}(\alpha, \beta)$ provided $|\beta| \geq \delta$.

The polynomials $N_{4}, N_{5}$ and $N_{6}$ have 599, 1625 and 3980 terms, respectively. The first few of their leading terms can be determined from Theorem 4 . 


$$
\begin{aligned}
N_{0}(\alpha, \beta)= & 1 \\
N_{1}(\alpha, \beta)= & 3 d^{2}|\beta|-8 d|\beta|+d \beta_{1}+|\beta| \alpha_{1}+|\beta| \beta_{1}+4|\beta|-\beta_{1}, \\
N_{2}(\alpha, \beta)= & \frac{9}{2} d^{4}|\beta|^{2}-\frac{9}{2} d^{4}|\beta|-24 d^{3}|\beta|^{2}+3 d^{3}|\beta| \beta_{1}+3 d^{2}|\beta|^{2} \alpha_{1}+3 d^{2}|\beta|^{2} \beta_{1} \\
& +24 d^{3}|\beta|-3 d^{3} \beta_{1}+23 d^{2}|\beta|^{2} \\
& -3 d^{2}|\beta| \alpha_{1}-14 d^{2}|\beta| \beta_{1}+\frac{1}{2} d^{2} \beta_{1}^{2}-8 d|\beta|^{2} \alpha_{1}-8 d|\beta|^{2} \beta_{1} \\
& +d|\beta| \alpha_{1} \beta_{1}+d|\beta| \beta_{1}^{2}+\frac{1}{2}|\beta|^{2} \alpha_{1}^{2}+|\beta|^{2} \alpha_{1} \beta_{1} \\
& +\frac{1}{2}|\beta|^{2} \beta_{1}^{2}-23 d^{2}|\beta|+\frac{21}{2} d^{2} \beta_{1}+\frac{3}{2} d|\beta|^{2}+8 d|\beta| \alpha_{1}+11 d|\beta| \beta_{1} \\
& +d|\beta| \beta_{2}-d \alpha_{1} \beta_{1}-\frac{5}{2} d \beta_{1}^{2}-\frac{1}{2}|\beta|^{2} \alpha_{1} \\
& +|\beta|^{2} \alpha_{2}-\frac{1}{2}|\beta|^{2} \beta_{1}+|\beta|^{2} \beta_{2}-\frac{1}{2}|\beta| \alpha_{1}^{2}-3|\beta| \alpha_{1} \beta_{1}-\frac{5}{2}|\beta| \beta_{1}^{2} \\
& -\frac{83}{2} d|\beta|-\frac{3}{2} d \beta_{1}-d \beta_{2}-48|\beta|^{2}+\frac{1}{2}|\beta| \alpha_{1} \\
& -|\beta| \alpha_{2}+\frac{29}{2}|\beta| \beta_{1}-3|\beta| \beta_{2}+2 \alpha_{1} \beta_{1}+3 \beta_{1}^{2}+48|\beta|-15 \beta_{1}+2 \beta_{2},
\end{aligned}
$$

$$
\begin{aligned}
N_{3}(\alpha, \beta)= & \frac{9}{2} d^{6}|\beta|^{3}-\frac{27}{2} d^{6}|\beta|^{2}-36 d^{5}|\beta|^{3}+\frac{9}{2} d^{5}|\beta|^{2} \beta_{1} \\
& +\frac{9}{2} d^{4}|\beta|^{3} \alpha_{1}+\frac{9}{2} d^{4}|\beta|^{3} \beta_{1}+9 d^{6}|\beta|+108 d^{5}|\beta|^{2}-\frac{27}{2} d^{5}|\beta| \beta_{1} \\
& +51 d^{4}|\beta|^{3}-\frac{27}{2} d^{4}|\beta|^{2} \alpha_{1}-42 d^{4}|\beta|^{2} \beta_{1}+\frac{3}{2} d^{4}|\beta| \beta_{1}^{2}-24 d^{3}|\beta|^{3} \alpha_{1} \\
& -24 d^{3}|\beta|^{3} \beta_{1}+3 d^{3}|\beta|^{2} \alpha_{1} \beta_{1}+3 d^{3}|\beta|^{2} \beta_{1}^{2} \\
& +\frac{3}{2} d^{2}|\beta|^{3} \alpha_{1}^{2}+3 d^{2}|\beta|^{3} \alpha_{1} \beta_{1}+\frac{3}{2} d^{2}|\beta|^{3} \beta_{1}^{2}-72 d^{5}|\beta|+9 d^{5} \beta_{1} \\
& -153 d^{4}|\beta|^{2}+9 d^{4}|\beta| \alpha_{1}+93 d^{4}|\beta| \beta 1-3 d^{4} \beta_{1}^{2} \\
& +\frac{1243}{6} d^{3}|\beta|^{3}+72 d^{3}|\beta|^{2} \alpha_{1}+92 d^{3}|\beta|^{2} \beta_{1}+3 d^{3}|\beta|^{2} \beta_{2}-9 d^{3}|\beta| \alpha_{1} \beta_{1} \\
& -\frac{35}{2} d^{3}|\beta| \beta_{1}^{2}+\frac{1}{6} d^{3} \beta_{1}^{3}+\frac{19}{2} d^{2}|\beta|^{3} \alpha_{1} \\
& +3 d^{2}|\beta|^{3} \alpha_{2}+\frac{19}{2} d^{2}|\beta|^{3} \beta_{1}+3 d^{2}|\beta|^{3} \beta_{2}-\frac{9}{2} d^{2}|\beta|^{2} \alpha_{1}^{2}-23 d^{2}|\beta|^{2} \alpha_{1} \beta_{1} \\
& -\frac{37}{2} d^{2}|\beta|^{2} \beta_{1}^{2}+\frac{1}{2} d^{2}|\beta| \alpha_{1} \beta_{1}^{2}
\end{aligned}
$$




$$
\begin{aligned}
& +\frac{1}{2} d^{2}|\beta| \beta_{1}^{3}-4 d|\beta|^{3} \alpha_{1}^{2}-8 d|\beta|^{3} \alpha_{1} \beta_{1}-4 d|\beta|^{3} \beta_{1}^{2}+\frac{1}{2} d|\beta|^{2} \alpha_{1}^{2} \beta_{1} \\
& +d|\beta|^{2} \alpha_{1} \beta_{1}^{2}+\frac{1}{2} d|\beta|^{2} \beta_{1}^{3}+\frac{1}{6}|\beta|^{3} \alpha_{1}^{3} \\
& +\frac{1}{2}|\beta|^{3} \alpha_{1}^{2} \beta_{1}+\frac{1}{2}|\beta|^{3} \alpha_{1} \beta_{1}^{2}+\frac{1}{6}|\beta|^{3} \beta_{1}^{3}+102 d^{4}|\beta|-54 d^{4} \beta_{1} \\
& -\frac{1243}{2} d^{3}|\beta|^{2}-48 d^{3}|\beta| \alpha_{1}-\frac{199}{2} d^{3}|\beta| \beta_{1} \\
& -9 d^{3}|\beta| \beta_{2}+6 d^{3} \alpha_{1} \beta_{1}+\frac{45}{2} d^{3} \beta_{1}^{2}-458 d^{2}|\beta|^{3}-\frac{57}{2} d^{2}|\beta|^{2} \alpha_{1} \\
& -9 d^{2}|\beta|^{2} \alpha_{2}+116 d^{2}|\beta|^{2} \beta_{1}-23 d^{2}|\beta|^{2} \beta_{2} \\
& +3 d^{2}|\beta| \alpha_{1}^{2}+\frac{95}{2} d^{2}|\beta| \alpha_{1} \beta_{1}+\frac{105}{2} d^{2}|\beta| \beta_{1}^{2}+d^{2}|\beta| \beta_{1} \beta_{2}-d^{2} \alpha_{1} \beta_{1}^{2} \\
& -2 d^{2} \beta_{1}^{3}+\frac{155}{2} d|\beta|^{3} \alpha_{1}-8 d|\beta|^{3} \alpha_{2} \\
& +\frac{155}{2} d|\beta|^{3} \beta_{1}-8 d|\beta|^{3} \beta_{2}+12 d|\beta|^{2} \alpha_{1}^{2}+\frac{61}{2} d|\beta|^{2} \alpha_{1} \beta_{1}+d|\beta|^{2} \alpha_{1} \beta_{2} \\
& +d|\beta|^{2} \alpha_{2} \beta_{1}+\frac{37}{2} d|\beta|^{2} \beta_{1}^{2}+2 d|\beta|^{2} \beta_{1} \beta_{2} \\
& -\frac{3}{2} d|\beta| \alpha_{1}^{2} \beta_{1}-\frac{11}{2} d|\beta| \alpha_{1} \beta_{1}^{2}-4 d|\beta| \beta_{1}^{3}-\frac{5}{2}|\beta|^{3} \alpha_{1}^{2}+|\beta|^{3} \alpha_{1} \alpha_{2} \\
& -5|\beta|^{3} \alpha_{1} \beta_{1}+|\beta|^{3} \alpha_{1} \beta_{2}+|\beta|^{3} \alpha_{2} \beta_{1}-\frac{5}{2}|\beta|^{3} \beta_{1}^{2} \\
& +|\beta|^{3} \beta_{1} \beta_{2}-\frac{1}{2}|\beta|^{2} \alpha_{1}^{3}-3|\beta|^{2} \alpha_{1}^{2} \beta_{1}-\frac{9}{2}|\beta|^{2} \alpha_{1} \beta_{1}^{2}-2|\beta|^{2} \beta_{1}^{3} \\
& +\frac{1243}{3} d^{3}|\beta|+\frac{70}{3} d^{3} \beta_{1}+6 d^{3} \beta_{2}+1374 d^{2}|\beta|^{2} \\
& +19 d^{2}|\beta| \alpha_{1}+6 d^{2}|\beta| \alpha_{2}-\frac{845}{2} d^{2}|\beta| \beta_{1}+48 d^{2}|\beta| \beta_{2}-27 d^{2} \alpha_{1} \beta_{1} \\
& -40 d^{2} \beta_{1}^{2}-2 d^{2} \beta_{1} \beta_{2}-\frac{842}{3} d|\beta|^{3} \\
& -\frac{465}{2} d|\beta|^{2} \alpha_{1}+24 d|\beta|^{2} \alpha_{2}-396 d|\beta|^{2} \beta_{1}+29 d|\beta|^{2} \beta_{2}+d|\beta|^{2} \beta_{3} \\
& -8 d|\beta| \alpha_{1}^{2}-33 d|\beta| \alpha_{1} \beta_{1}-3 d|\beta| \alpha_{1} \beta_{2} \\
& -3 d|\beta| \alpha_{2} \beta_{1}+2 d|\beta| \beta_{1}^{2}-11 d|\beta| \beta_{1} \beta_{2}+d \alpha_{1}^{2} \beta_{1}+7 d \alpha_{1} \beta_{1}^{2}+\frac{47}{6} d \beta_{1}^{3} \\
& -\frac{92}{3}|\beta|^{3} \alpha_{1}-6|\beta|^{3} \alpha_{2}+|\beta|^{3} \alpha_{3}
\end{aligned}
$$




$$
\begin{aligned}
& -\frac{92}{3}|\beta|^{3} \beta_{1}-6|\beta|^{3} \beta_{2}+|\beta|^{3} \beta_{3}+\frac{15}{2}|\beta|^{2} \alpha_{1}^{2}-3|\beta|^{2} \alpha_{1} \alpha_{2} \\
& +\frac{87}{2}|\beta|^{2} \alpha_{1} \beta_{1}-6|\beta|^{2} \alpha_{1} \beta_{2}-6|\beta|^{2} \alpha_{2} \beta_{1}+36|\beta|^{2} \beta_{1}^{2} \\
& -9|\beta|^{2} \beta_{1} \beta_{2}+\frac{1}{3}|\beta| \alpha_{1}^{3}+\frac{11}{2}|\beta| \alpha_{1}^{2} \beta_{1}+13|\beta| \alpha_{1} \beta_{1}^{2}+\frac{47}{6}|\beta| \beta_{1}^{3} \\
& -916 d^{2}|\beta|+303 d^{2} \beta_{1}-28 d^{2} \beta_{2}+842 d|\beta|^{2} \\
& +155 d|\beta| \alpha_{1}-16 d|\beta| \alpha_{2}+\frac{1237}{2} d|\beta| \beta_{1}-31 d|\beta| \beta_{2}-3 d|\beta| \beta_{3} \\
& +8 d \alpha_{1} \beta_{1}+2 d \alpha_{1} \beta_{2}+2 d \alpha_{2} \beta_{1}-\frac{103}{2} d \beta_{1}^{2}+14 d \beta_{1} \beta_{2}+706|\beta|^{3} \\
& +92|\beta|^{2} \alpha_{1}+18|\beta|^{2} \alpha_{2}-3|\beta|^{2} \alpha_{3}-46|\beta|^{2} \beta_{1}+48|\beta|^{2} \beta_{2} \\
& -6|\beta|^{2} \beta_{3}-5|\beta| \alpha_{1}^{2}+2|\beta| \alpha_{1} \alpha_{2}-\frac{197}{2}|\beta| \alpha_{1} \beta_{1}+11|\beta| \alpha_{1} \beta_{2} \\
& +11|\beta| \alpha_{2} \beta_{1}-\frac{271}{2}|\beta| \beta_{1}^{2}+26|\beta| \beta_{1} \beta_{2}-3 \alpha_{1}^{2} \beta_{1}-12 \alpha_{1} \beta_{1}^{2}-10 \beta_{1}^{3} \\
& -\frac{1684}{3} d|\beta|-\frac{808}{3} d \beta_{1}+10 d \beta_{2}+2 d \beta_{3}-2118|\beta|^{2} \\
& -\frac{184}{3}|\beta| \alpha_{1}-12|\beta| \alpha_{2}+2|\beta| \alpha_{3}+\frac{1184}{3}|\beta| \beta_{1}-102|\beta| \beta_{2} \\
& +11|\beta| \beta_{3}+63 \alpha_{1} \beta_{1}-6 \alpha_{1} \beta_{2}-6 \alpha_{2} \beta_{1}+150 \beta_{1}^{2}-24 \beta_{1} \beta_{2} \\
& +1412|\beta|-362 \beta_{1}+60 \beta_{2}-6 \beta_{3} \text {. }
\end{aligned}
$$

\section{References}

1. Ardila, F., Block, F.: Universal polynomials for Severi degrees of toric surfaces. Preprint (2010). arXiv:1012.5305

2. Block, F.: Relative node polynomials for plane curves. Preprint (2010). arXiv:1009.5063

3. Block, F.: Computing node polynomials for plane curves. Math. Res. Lett. 18, 621-643 (2011)

4. Block, F., Gathmann, A., Markwig, H.: Psi-floor diagrams and a Caporaso-Harris type recursion. Israel J. Math. (to appear, 2011)

5. Brugallé, E., Mikhalkin, G.: Enumeration of curves via floor diagrams. C. R. Math. Acad. Sci. Paris 345(6), 329-334 (2007)

6. Brugallé, E., Mikhalkin, G.: Floor decompositions of tropical curves: the planar case. In: Proceedings of Gökova Geometry-Topology Conference (GGT), Gökova, 2008, pp. 64-90 (2009)

7. Caporaso, L., Harris, J.: Counting plane curves of any genus. Invent. Math. 131(2), 345-392 (1998)

8. Di Francesco, P., Itzykson, C.: Quantum intersection rings. In: The Moduli Space of Curves, Texel Island, 1994. Progr. Math., vol. 129, pp. 81-148. Birkhäuser Boston, Boston (1995)

9. Enriques, F.: Sui moduli d'una classe di superficie e sul teorema d'esistenza per funzioni algebriche di due variabilis. Atti R. Accad. Sci. Torino 47 (1912)

10. Fomin, S., Mikhalkin, G.: Labeled floor diagrams for plane curves. J. Eur. Math. Soc. (JEMS) 12(6), 1453-1496 (2010)

11. Gathmann, A.: Tropical algebraic geometry. Jahresber. Dtsch. Math.-Ver. 108(1), 3-32 (2006) 
12. Göttsche, L.: A conjectural generating function for numbers of curves on surfaces. Commun. Math. Phys. 196(3), 523-533 (1998)

13. Graham, R.L., Knuth, D.E., Patashnik, O.: Concrete Mathematics, 2nd edn. Addison-Wesley, Reading (1994). A foundation for computer science

14. Harris, J.: On the Severi problem. Invent. Math. 84(3), 445-461 (1986)

15. Kleiman, S., Piene, R.: Node polynomials for families: methods and applications. Math. Nachr. 271, 69-90 (2004)

16. Mikhalkin, G.: Enumerative tropical geometry in $\mathbb{R}^{2}$. J. Am. Math. Soc. 18, 313-377 (2005)

17. Qviller, N.: The Di Francesco-Itzykson-Göttsche conjectures for node polynomials of $\mathbb{P}^{2}$. Int. J. Math. (2010). doi:10.1142/S0129167X12500498. arXiv:1010.2377

18. Richter-Gebert, J., Sturmfels, B., Theobald, T.: First steps in tropical geometry. In: Idempotent Mathematics and Mathematical Physics. Contemp. Math., vol. 377, pp. 289-317. Am. Math. Soc, Providence (2005)

19. Severi, F.: Vorlesungen über Algebraische Geometrie. Teubner, Leipzig (1921)

20. Speyer, D., Sturmfels, B.: The tropical Grassmannian. Adv. Geom. 4(3), 389-411 (2004)

21. Stanley, R.: Enumerative Combinatorics, Vol. 1. Cambridge Studies in Advanced Mathematics, vol. 49. Cambridge University Press, Cambridge (1997)

22. Tzeng, Y.-J.: A proof of Göttsche-Yau-Zaslow formula. Preprint (2010). arXiv:1009.5371

23. Vainsencher, I.: Enumeration of $n$-fold tangent hyperplanes to a surface. J. Algebr. Geom. 4(3), 503526 (1995)

24. Vakil, R.: Counting curves on rational surfaces. Manuscr. Math. 102(1), 53-84 (2000) 